\title{
Cross Metathesis as a General Strategy for the Synthesis of Prostacyclin and Prostaglandin Analogues - Supporting Information
}

Neil A. Sheddan and Johann Mulzer*

Institut für Organische Chemie, Währingerstrasse 38, A-1090 Wien, Austria

General Experimental

All reactions were carried out in oven or flame-dried glassware under an argon atmosphere, unless otherwise stated. Anhydrous tetrahydrofuran (THF) and diethyl ether $\left(\mathrm{Et}_{2} \mathrm{O}\right)$ were freshly distilled from sodium / benzophenone under argon. All other solvents were HPLC grade. Reactions were magnetically stirred and monitored by thin layer chromatography (TLC) with E. Merck silica gel 60-F254 plates. Flash column chromatography was performed with Merck silica gel (0.04-0.63 $\mu \mathrm{m}, 240-400 \mathrm{mesh})$ under high pressure. Yields refer to chromatographically and spectroscopically pure compounds, unless otherwise stated. NMR spectra were recorded on either a Bruker Avance DPX $400 \mathrm{MHz}$ or $600 \mathrm{MHz}$ spectrometer. Unless otherwise stated, all NMR spectra were measured in $\mathrm{CDCl}_{3}$ solutions and referenced to the residual $\mathrm{CHCl}_{3}$ signal $\left({ }^{1} \mathrm{H}, \delta=7.26 ;{ }^{13} \mathrm{C}, \delta=77.0\right)$. All ${ }^{1} \mathrm{H}$ and ${ }^{13} \mathrm{C}$ NMR shifts are given in ppm (for ${ }^{1} \mathrm{H}$ NMR: $\mathrm{s}=$ singlet, $\mathrm{d}=$ doublet, $\mathrm{t}=$ triplet, $\mathrm{q}=$ quadruplet, $\mathrm{m}=$ multiplet; br $\mathrm{s}=$ broad signal; for ${ }^{13} \mathrm{C}$ NMR: $\mathrm{p}=$ primary, $\mathrm{s}=$ secondary, $\mathrm{t}=$ tertiary, $\mathrm{q}=$ quaternary). Coupling constants $J$ are given in Hz; assignments of proton resonances were confirmed, when possible, by selective homonuclear decoupling experiments or by correlated spectroscopy.

Reference [7h] in full: T. Maruyama, S.-I. Kuwabe, Y. Kawanaka, T. Shiraishi, Y. Shinagawa, K. Sakata, A. Seki, Y. Kishida, H. Yoshida, T. Maruyama, Bioorg. Med. Chem. 2002, 10, 2103-2110. 


\section{(S)-tert-butyldimethyl(oct-1-en-3-yloxy)silane 9}

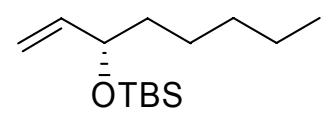

9

$\mathrm{C}_{14} \mathrm{H}_{30} \mathrm{OSi} \quad \mathrm{M}_{\mathrm{r}}=242.47$

${ }^{1} \mathbf{H}$ NMR $\left(400 \mathrm{MHz}, \mathrm{CDCl}_{3}\right) 5.80\left(1 \mathrm{H}, \mathrm{ddd}, J 17.2,10.4,6.1, \mathrm{H}_{2} \mathrm{C}=\mathrm{CH}\right), 5.13(1 \mathrm{H}, \mathrm{ddd}, J$ 17.2, 1.9, 1.3, $H \mathrm{HC}=\mathrm{CH}), 5.01(1 \mathrm{H}, \mathrm{ddd}, J 10.4,1.9,1.1, \mathrm{H} H \mathrm{C}=\mathrm{CH}), 4.08(1 \mathrm{H}$, dddd, $J 12.1$, $6.1,1.2,1.2, \mathrm{CHOTBS}), \quad 1.57-1.41\left(2 \mathrm{H}, \mathrm{m}, \mathrm{TBSOCHCH}{ }_{2}\right), 1.41-1.20(6 \mathrm{H}, \mathrm{m}$, $\left.\mathrm{CH}_{2} \mathrm{CH}_{2} \mathrm{CH}_{2} \mathrm{CH}_{3}\right), 0.93-0.85\left(3 \mathrm{H}, \mathrm{m}, \mathrm{CH}_{3}\right), 0.90\left(9 \mathrm{H}, \mathrm{s}, \mathrm{SiC}\left(\mathrm{CH}_{3}\right)_{3}\right), 0.06\left(3 \mathrm{H}, \mathrm{s}, \mathrm{SiCH}_{3}\right), 0.04$ $\left(3 \mathrm{H}, \mathrm{s}, \mathrm{SiCH}_{3}\right)$

${ }^{13}$ C-NMR (100 MHz, $\left.\mathrm{CDCl}_{3}\right) 141.9$ (t), 113.3 (s), 73.9 (t), 38.1 (s), 31.8 (s), 25.9 (p), 24.8 (s), $22.6(\mathrm{~s}), 18.2(\mathrm{q}), 14.0(\mathrm{p}),-4.4(\mathrm{p}),-4.8(\mathrm{p})$

IR (Si, Film) $\widetilde{v}_{\max }=2957,2930,2858,1472,1252,1081,920,836$

HRMS $\left(70{ }^{\circ} \mathrm{C} 70 \mathrm{eV}\right): \mathrm{m} / z$ calcd for $\mathrm{C}_{14} \mathrm{H}_{30} \mathrm{OSi}$ : 242.2066; found 242.2059

Optical Rotation: $[\alpha]_{\mathrm{D}}^{20}=+6.5(\mathrm{c}=1.09$, acetone $)$

$\boldsymbol{R}_{\mathbf{f}}(10: 1 \mathrm{Hex} /$ EtOAc $)=0.68$

\section{$\underline{\text { (R)-tert-butyldimethyl(1-m-tolylbut-3-en-2-yloxy)silane } 12}$}

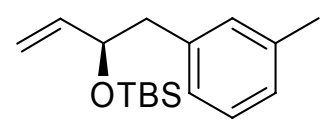

12

$\mathrm{C}_{17} \mathrm{H}_{28} \mathrm{O}_{2} \mathrm{Si} \quad \mathbf{M}_{\mathbf{r}}=276.49$

${ }^{1} \mathbf{H}$ NMR $\left(400 \mathrm{MHz}, \mathrm{CDCl}_{3}\right) 7.17(1 \mathrm{H}, \mathrm{dd}, J$ 7.8, 7.8, $\mathrm{Ar} H)$, 7.05-6.97 (3H, m, ArH), 5.88 $\left(1 \mathrm{H}, \mathrm{ddd}, J 17.2,10.3,5.8, \mathrm{H}_{2} \mathrm{C}=\mathrm{CH}\right), 5.18(1 \mathrm{H}, \mathrm{ddd}, J 17.2,1.6,1.6, H \mathrm{HC}=\mathrm{CH}) 5.05(1 \mathrm{H}$, ddd, $J 10.3,1.6,1.6, \mathrm{H} H \mathrm{C}=\mathrm{CH}),(1 \mathrm{H}, \mathrm{dddd}, J 6.1,5.8,1.2,1.2, \mathrm{CHOTBS}), 2.74(2 \mathrm{H}, \mathrm{d}, J 6.8$, 
$\left.\left.\mathrm{CH}_{2} \mathrm{ArCH}_{3}\right), 2.34(3 \mathrm{H}, \mathrm{s}, \mathrm{ArCH})_{3}\right), 0.86\left(9 \mathrm{H}, \mathrm{s}, \mathrm{SiC}\left(\mathrm{CH}_{3}\right)_{3}\right),-0.08(3 \mathrm{H}, \mathrm{s}, \mathrm{SiCH}),-0.20(3 \mathrm{H}, \mathrm{s}$, $\left.\mathrm{SiCH}_{3}\right)$

${ }^{13}$ C-NMR (100 MHz, $\left.\mathrm{CDCl}_{3}\right) 141.3$ (t), 138.6 (q), 137.4 (q), 130.9 (t), 127.9 (t), 126.8 (t), $126.8(\mathrm{t}), 113.6(\mathrm{~s}), 75.1(\mathrm{t}), 45.0(\mathrm{~s}), 25.8(\mathrm{p}), 21.3(\mathrm{p}), 18.2(\mathrm{q}),-4.8(\mathrm{p}),-5.3(\mathrm{p})$

IR (Si, Film) $\widetilde{v}_{\max }=3024,2956,2928,2857,1644,1610,1472,1462,1124,1078,1034$

HRMS $\left(70{ }^{\circ} \mathrm{C} 70 \mathrm{eV}\right): \mathrm{m} / z$ calcd for $\mathrm{C}_{17} \mathrm{H}_{28} \mathrm{OSi}$ : 276.1909; found 276.1900

Optical Rotation: $[\alpha]_{\mathrm{D}}^{20}=+3.5(\mathrm{c}=1.12$, acetone $)$

$\boldsymbol{R}_{\mathbf{f}}(10: 1 \mathrm{Hex} /$ EtOAc $)=0.68$

tert-butyl((2R,3R,3aS,6aS)-5-(5-(4-methoxybenzyloxy)pentyl)-3-vinyl-1,2,3,3a,4,6ahexahydropentalen-2-yloxy)dimethylsilane 16a

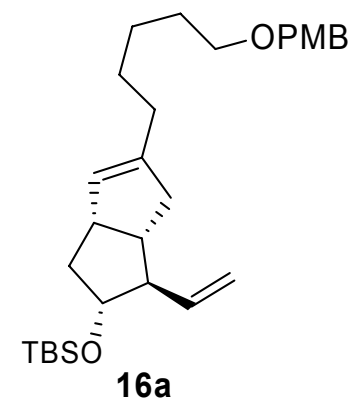

$\mathrm{C}_{29} \mathrm{H}_{46} \mathrm{O}_{3} \mathrm{Si} \quad \mathrm{M}_{\mathrm{r}}=470.76$

${ }^{1} \mathbf{H}$ NMR $\left(400 \mathrm{MHz}, \mathrm{CDCl}_{3}\right) 7.26(2 \mathrm{H}, \mathrm{d}, J$ 8.6, $\mathrm{ArH}), 6.88(2 \mathrm{H}, \mathrm{d}, J$ 8.6, $\mathrm{Ar} H), 5.71(1 \mathrm{H}$, $\left.\mathrm{ddd}, J 17.2,10.1,7.8, \mathrm{H}_{2} \mathrm{C}=\mathrm{CH}\right), 5.24(1 \mathrm{H}, \mathrm{d}, J 1.5$, vinylic in ring), 5.10-4.98 $(2 \mathrm{H}, \mathrm{m}$, $\left.H_{2} \mathrm{C}=\mathrm{CH}\right) .4 .43\left(2 \mathrm{H}, \mathrm{s}, \mathrm{OCH}_{2} \mathrm{Ar}\right), 3.80\left(3 \mathrm{H}, \mathrm{s}, \mathrm{ArOCH}_{3}\right), 3.74(1 \mathrm{H}, \mathrm{ddd}, J$ 9.6, 9.6, 6.8, CHOTBS), 3.44 (2H, dd, $J$ 6.7, 6.7, alkyl $\mathrm{CH}_{2}$ ), 2.94 (1H, pseudo q, $J$ 9.0, $\mathrm{CHCH}_{2} \mathrm{CHOTBS}$ ), $2.42(1 \mathrm{H}, \mathrm{dd}, J 16.3,8.7, \mathrm{HC}=\mathrm{CC} H \mathrm{H}), 2.28\left(1 \mathrm{H}, \mathrm{dddd}, J 9.5,9.5,8.8,1.7, \mathrm{H}_{2} \mathrm{C}=\mathrm{CHCHCH}\right)$, 2.19 (1H, ddd, $J$ 12.1, 8.8, 7.1, CHHCHOTBS), 2.05-1.93 (4H, m, CHCHOTBS, HC=CCH , alkyl $\left.\mathrm{CH}_{2}\right), 1.66-1.56\left(2 \mathrm{H}, \mathrm{m}\right.$, alkyl $\left.\mathrm{CH}_{2}\right), 1.48-1.22\left(7 \mathrm{H}, \mathrm{m}, \mathrm{CHHCHOTBS}, 3 \mathrm{x}\right.$ alkyl $\left.\mathrm{CH}_{2}\right)$, $0.87\left(9 \mathrm{H}, \mathrm{s}, \mathrm{SiC}\left(\mathrm{CH}_{3}\right)_{3}\right), 0.02\left(6 \mathrm{H}, \mathrm{s}, \mathrm{Si}\left(\mathrm{CH}_{3}\right)_{2}\right)$ 
${ }^{13}$ C-NMR (100 MHz, $\left.\mathrm{CDCl}_{3}\right) 159.1$ (q), 141.8 (q), 140.4 (t), 130.8 (q), 129.2 (t), 127.8 (t), $115.3(\mathrm{~s}), 113.7(\mathrm{t}), 77.7(\mathrm{t}), 72.5(\mathrm{~s}), 70.1(\mathrm{~s}), 58.9(\mathrm{t}), 55.2(\mathrm{p}), 45.4(\mathrm{~s}), 43.0(\mathrm{~s}), 40.7(\mathrm{~s})$, 40.0 (s), 31.0 (s), 29.6 (s), 27.6 (s), 26.1 (s), 25.8 (p), 18.1 (q), -4.5 (p), -4.6 (p)

IR (Si, Film) $\widetilde{v}_{\max }=2929,2855,1640,1613,1585,1512,1462,1360,1248,1171,1039$

HRMS $\left(100{ }^{\circ} \mathrm{C} 70 \mathrm{eV}\right): \mathrm{m} / z$ calcd for $\mathrm{C}_{29} \mathrm{H}_{46} \mathrm{O}_{3} \mathrm{Si}: 470.3216$; found 470.3229

Optical Rotation: $[\alpha]_{\mathrm{D}}^{20}=-16.3(\mathrm{c}=0.83$, acetone $)$

$\boldsymbol{R}_{\mathbf{f}}(3: 1 \mathrm{Hex} / \mathrm{EtOAc})=0.58$

\section{$\underline{(3 a R, 4 R, 5 R, 6 a S)-5-(t e r t-b u t y l d i m e t h y l s i l y l o x y)-4-v i n y l h e x a h y d r o-2 H-~}$} cyclopenta $[b]$ furan-2-one, 22a

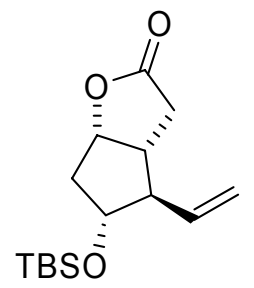

$22 a$

$\mathrm{C}_{15} \mathrm{H}_{26} \mathrm{O}_{3} \mathrm{Si} \quad \mathrm{M}_{\mathrm{r}}=282.45$

${ }^{1} \mathbf{H}$ NMR (400 MHz, $\left.\mathrm{CDCl}_{3}\right) 5.62\left(1 \mathrm{H}, \mathrm{ddd}, J\right.$ 16.7, 10.8, 7.6, $\left.\mathrm{H}_{2} \mathrm{C}=\mathrm{CH}\right), 5.12-5.10(1 \mathrm{H}, \mathrm{m}$, $H \mathrm{HC}=\mathrm{CH}), 5.07(1 \mathrm{H}, \mathrm{ddd}, J 8.1,1.3,1.3, \mathrm{H} H \mathrm{C}=\mathrm{CH}), 4.93(1 \mathrm{H}, \mathrm{ddd}, J 7.1,7.1,2.5$, HCOC $=\mathrm{O}), 3.98$ (1H, ddd, $J$ 6.1, 5.9, 5.8, CHOTBS), 2.75 (1H, dd, J 17.4, 10.1, $\mathrm{OC}=\mathrm{OCH}), 2.70-2.62\left(1 \mathrm{H}, \mathrm{m}, \mathrm{H}_{2} \mathrm{C}=\mathrm{CHCHCH}\right), 2.49(1 \mathrm{H}, \mathrm{dd}, J 17.7,2.3, \mathrm{OC}=\mathrm{OCH} H)$, $2.42\left(1 \mathrm{H}, \mathrm{dd}, J 13.4,6.8, \mathrm{H}_{2} \mathrm{C}=\mathrm{CHCH}\right), 2.29$ (1H, ddd, $J$ 14.6, 7.1, 6.1, CHHCHOTBS), 1.97 (1H, ddd, J 14.7, 5.7, 2.6, CHHCHOTBS), 0.87 (9H, s, SiC(CH3) $\left.)_{3}\right), 0.04$ (3H, s, $\left.\mathrm{SiCH}_{3}\right), 0.04$ $\left(3 \mathrm{H}, \mathrm{s}, \mathrm{SiCH}_{3}\right)$

${ }^{13}$ C-NMR (100 MHz, $\left.\mathrm{CDCl}_{3}\right) 177.0$ (q), 137.6 (t), 116.7 (s), 83.1 (t), 77.7 (t), 58.0 (t), 41.7 (t), $40.6(\mathrm{~s}), 34.8(\mathrm{~s}), 25.7(\mathrm{p}), 18.0(\mathrm{q}),-4.8(\mathrm{p}),-4.9(\mathrm{p})$

IR (Si, Film) $\widetilde{v}_{\max }=2930,2857,1760,1644,1471,1360,1258,1168,1127,1036,980$ 
HRMS $\left(100{ }^{\circ} \mathrm{C} 70 \mathrm{eV}\right): \mathrm{m} / z$ calcd for $\mathrm{C}_{15} \mathrm{H}_{26} \mathrm{O}_{3} \mathrm{Si}: 282.1651$; found 282.1649

Optical Rotation: $[\alpha]_{\mathrm{D}}^{20}=-23.5(\mathrm{c}=0.31$, acetone $)$

$\boldsymbol{R}_{\mathbf{f}}(1: 2 \mathrm{Hex} / \mathrm{EtOAc})=0.65$

$\underline{(3 a S, 4 R, 5 R, 6 a R)-5-(t e r t-b u t y l d i m e t h y l s i l y l o x y)-4-v i n y l h e x a h y d r o p e n t a l e n-2(1 H)-o n e, ~} 23$

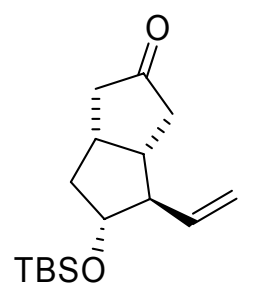

23

$\mathrm{C}_{16} \mathrm{H}_{28} \mathrm{O}_{2} \mathrm{Si} \quad \mathrm{M}_{\mathrm{r}}=280.48$

${ }^{1} \mathbf{H}$ NMR (400 MHz, $\left.\mathrm{CDCl}_{3}\right) 5.67\left(1 \mathrm{H}\right.$, ddd, $J$ 17.7, 9.6, 8.1, $\left.\mathrm{H}_{2} \mathrm{C}=\mathrm{CH}\right)$, 5.09-5.05 (1H, m, $H \mathrm{HC}=\mathrm{CH}), 5.05-5.02(1 \mathrm{H}, \mathrm{m}, \mathrm{H} H \mathrm{C}=\mathrm{CH}), 3.94(1 \mathrm{H}, \mathrm{ddd}, J$ 7.3, 7.1, 7.1, CHOTBS $), 2.76-$ $2.65\left(1 \mathrm{H}, \mathrm{m}, \mathrm{CHCH}_{2} \mathrm{CHOTBS}\right), 2.56$ (1H, ddd, $J$ 19.0, 10.4, 1.3, $\left.\mathrm{O}=\mathrm{CCHHCHCH}\right), 2.52-2.42$ $\left(2 \mathrm{H}, \mathrm{m}, \mathrm{CHCHCH}=\mathrm{CH}_{2}, \mathrm{O}=\mathrm{CC} H \mathrm{HCHCH}_{2}\right), 2.34$ (1H, ddd, $J$ 13.1, 6.3, 1.6, CHHCHOTBS), 2.26-2.11 (3H, m, CHCHOTBS, $\left.\mathrm{O}=\mathrm{CCH} H \mathrm{CHCH}, \mathrm{O}=\mathrm{CCH} H \mathrm{CHCH}_{2}\right), 1.47(1 \mathrm{H}, \mathrm{ddd}, J$ 13.2, 7.0, 6.9, CHHCHOTBS), $0.86\left(9 \mathrm{H}, \mathrm{s}, \mathrm{SiC}\left(\mathrm{CH}_{3}\right)_{3}\right), 0.03\left(6 \mathrm{H}, \mathrm{s}, \mathrm{Si}\left(\mathrm{CH}_{3}\right)_{2}\right)$

${ }^{13}$ C-NMR (100 MHz, $\left.\mathrm{CDCl}_{3}\right) 220.1$ (q), 139.1 (t), 116.1 (s), 78.9 (t), 59.2 (t), 45.9 (s), 43.1 $(\mathrm{s}), 42.4(\mathrm{t}), 42.3(\mathrm{~s}), 35.5(\mathrm{t}), 25.8(\mathrm{p}), 18.1(\mathrm{q}),-4.7(\mathrm{p})$

IR (Si, Film) $\widetilde{v}_{\max }=2955,2930,2857,1742,1641,1472,1407,1257,1122,1004$

HRMS $\left(100{ }^{\circ} \mathrm{C} 70 \mathrm{eV}\right): \mathrm{m} / z$ calcd for $\mathrm{C}_{16} \mathrm{H}_{28} \mathrm{O}_{2} \mathrm{Si}: 280.1859$; found 280.1848

Optical Rotation: $[\alpha]_{\mathrm{D}}^{20}=-30.7(\mathrm{c}=0.5$, acetone $)$

$\mathbf{R}_{\mathbf{f}}(3: 1 \mathrm{Hex} /$ EtOAc $)=0.46$ 


\section{General Procedure for Cross Metathesis (CM) for Isocarbacyclin Derivatives}

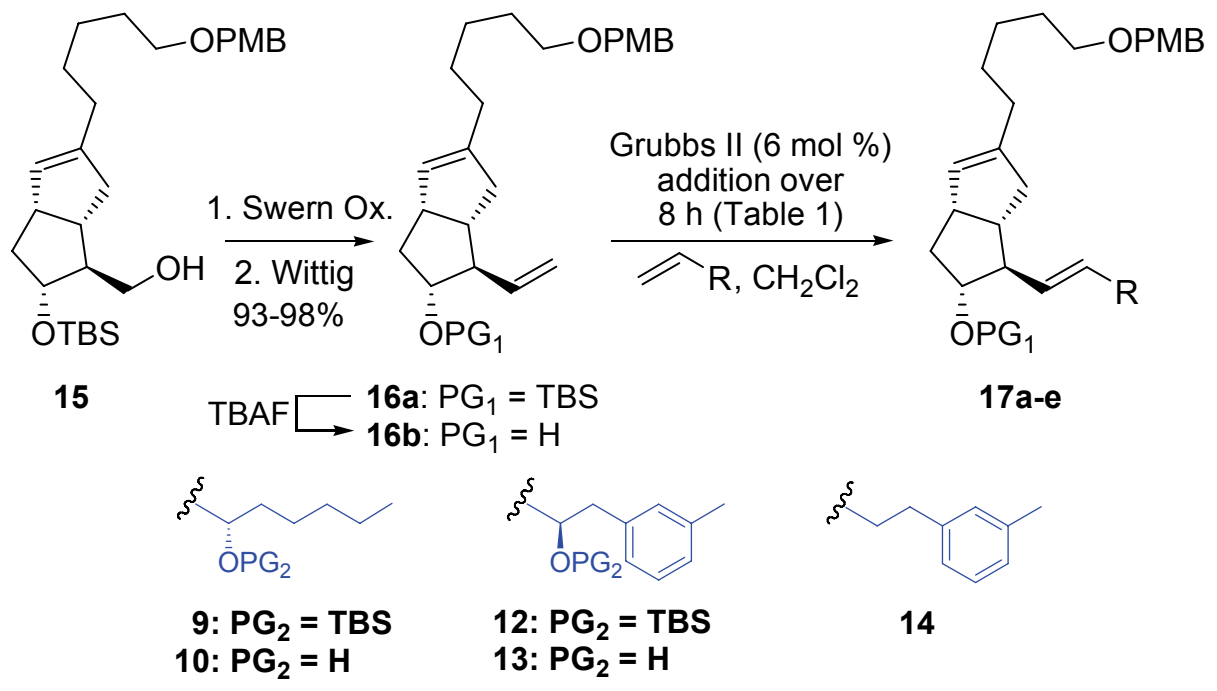

$\underline{\text { Table } 1 \text { Isocarbacyclin Derivative Cross Metathesis (Yields Not Optimized) }}{ }^{\mathrm{a}}$

\begin{tabular}{|c|c|c|c|c|c|c|}
\hline entry & $\begin{array}{l}\text { bicyclic } \\
\text { olefin }\end{array}$ & $\begin{array}{l}\omega \text {-side chain } \\
\text { olefin } \mathrm{R}^{\mathrm{b}}\end{array}$ & $\mathrm{PG}_{1}$ & product & $E: Z$ & $\begin{array}{l}\text { yield } \\
(\%)^{[c]}\end{array}$ \\
\hline 1 & $16 a$ & & TBS & $17 \mathbf{a}$ & $15: 1$ & 82 \\
\hline 2 & $16 a$ & & TBS & $17 b$ & $11: 1$ & $61^{\mathrm{d}}$ \\
\hline 3 & $16 \mathrm{~b}$ & & $\mathrm{H}$ & $17 \mathrm{c}$ & $13: 1$ & 74 \\
\hline 4 & $16 a$ & & TBS & 17d & $19: 1$ & 90 \\
\hline 5 & $16 a$ & & TBS & $17 \mathrm{e}$ & $16: 1$ & $66^{\mathrm{d}}$ \\
\hline
\end{tabular}

${ }^{\mathrm{a}}$ All reactions were carried out in $\mathrm{CH}_{2} \mathrm{Cl}_{2}$ at $40{ }^{\circ} \mathrm{C} .{ }^{\mathrm{b}} 2$ equivalents of $\omega$-side chain used. Isolated yields. ${ }^{\mathrm{d}}$ 30-35\% homodimerized $\omega$-side chain recovered.

The following procedure for the preparation of tert-butyl $((2 R, 3 R, 3 a S, 6 a S)-3-((R, E)-3-(t e r t-$ butyldimethylsilyloxy)-4-m-tolylbut-1-enyl)-5-(5-(4-methoxybenzyloxy)pentyl)-

1,2,3,3a,4,6a-hexahydropentalen-2-yloxy)dimethylsilane, $\mathbf{1 7 d}$, is representative.

To a solution of bicyclic olefin $16 \mathbf{a}(0.250 \mathrm{~g}, 0.53 \mathrm{mmol})$ and $\omega$-side chain olefin $12(0.293 \mathrm{~g}$, $1.06 \mathrm{mmol})$ in degassed $\mathrm{CH}_{2} \mathrm{Cl}_{2}$ or degassed $\mathrm{C}_{6} \mathrm{H}_{6} \mathrm{Me}^{*}(30 \mathrm{~mL})$ was added a solution of Grubbs II (6 mol \%, $0.027 \mathrm{~g}, 0.032 \mathrm{mmol})$ in degassed $\mathrm{CH}_{2} \mathrm{Cl}_{2}$ or degassed $\mathrm{C}_{6} \mathrm{H}_{6} \mathrm{Me}(7 \mathrm{~mL})$ via syringe pump (over $8 \mathrm{hr}$.) at 40 or $80^{\circ} \mathrm{C}$ (see Table 1). After the reaction was complete (monitored by TLC), air was bubbled through the solution to destroy the catalyst. The mixture was concentrated in vacuo and purified by flash column chromatography using a 
hexanes:ethyl acetate mixture as the mobile phase to afford the corresponding cross metathesis product.

* Degassing of $\mathrm{CH}_{2} \mathrm{Cl}_{2}$ and $\mathrm{C}_{6} \mathrm{H}_{6} \mathrm{Me}$ was achieved by carrying out 4 cycles of the pump and freeze technique.

tert-butyl $((S, E)-1-((1 R, 2 R, 3 a S, 6 a S)-2-($ tert-butyldimethylsilyloxy)-5-(5-(4methoxybenzyloxy)pentyl)-1,2,3,3a,6,6a-hexahydropentalen-1-yl)oct-1-en-3yloxy)dimethylsilane, 17 a

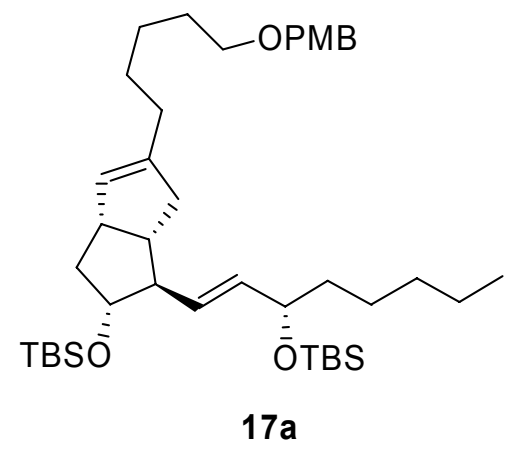

$\mathbf{C}_{41} \mathbf{H}_{72} \mathbf{O}_{4} \mathbf{S i}_{2} \quad \mathbf{M}_{\mathbf{r}}=685.18$

${ }^{1}$ H NMR (600 MHz, $\left.\mathrm{CDCl}_{3}\right) 7.26(2 \mathrm{H}, \mathrm{d}, J$ 8.5, $\mathrm{ArH}), 6.88(2 \mathrm{H}, \mathrm{d}, J$ 8.5, $\mathrm{ArH}), 5.52-5.44$ $(1 \mathrm{H}$, overlapping dd, $J 15.4, H \mathrm{C}=\mathrm{CH}), 5.52-5.44(1 \mathrm{H}$, overlapping dd, $J 15.4, \mathrm{HC}=\mathrm{CH}), 5.23$ $\left(1 \mathrm{H}, \mathrm{d}, J 1.5\right.$, vinylic in ring), $4.43\left(2 \mathrm{H}, \mathrm{s}, \mathrm{OCH} \mathrm{H}_{2} \mathrm{Ar}\right), 4.06(1 \mathrm{H}, \mathrm{ddd}, J 6.2,6.2,4.7$, $\mathrm{HC}=\mathrm{CHCHOTBS}), 3.80\left(3 \mathrm{H}, \mathrm{s}, \mathrm{ArOCH}_{3}\right), 3.74(1 \mathrm{H}, \mathrm{ddd}, J$ 9.4, 7.0, 7.0, CHOTBS $), 3.44$ (2H, dd, $J$ 6.8, 6.6, alkyl $\left.\mathrm{CH}_{2}\right), 2.97-2.88$ (1H, m, $\left.\mathrm{CHCH}_{2} \mathrm{CHOTBS}\right), 2.39$ (1H, dd, $J$ 16.3, 8.6, $\mathrm{HC}=\mathrm{CCHH}), 2.23(1 \mathrm{H}, \mathrm{dddd}, J 9.4,9.4,8.8,1.7, H \mathrm{CHCHC}=\mathrm{CH}), 2.18(1 \mathrm{H}$, ddd, $J 12.2$, 8.8, 6.9, CHHCHOTBS), 2.04-1.93 (4H, m, $H \mathrm{CHC}=\mathrm{CH}, \mathrm{HC}=\mathrm{CCH} H$, alkyl $\left.\mathrm{CH}_{2}\right), 1.61(2 \mathrm{H}$, dddd, $J$ 7.5, 7.3, 6.8, 6.8, alkyl $\left.\mathrm{CH}_{2}\right), 1.53-1.38\left(4 \mathrm{H}, \mathrm{m}, 2 \mathrm{x}\right.$ alkyl $\left.\mathrm{CH}_{2}\right), 1.38-1.22(9 \mathrm{H}, 4 \mathrm{x}$ $\left.\mathrm{CH}_{2}, \mathrm{CH} H \mathrm{CHOTBS}\right), 0.90-0.88\left(3 \mathrm{H}, \mathrm{m}\right.$, alkyl $\left.\mathrm{CH}_{3}\right), 0.89\left(9 \mathrm{H}, \mathrm{s}, \mathrm{SiC}\left(\mathrm{CH}_{3}\right)_{3}\right), 0.86(9 \mathrm{H}, \mathrm{s}$, $\left.\mathrm{SiC}\left(\mathrm{CH}_{3}\right)_{3}\right), 0.04\left(3 \mathrm{H}, \mathrm{s}, \mathrm{SiCH}_{3}\right), 0.02\left(9 \mathrm{H}, \mathrm{s}, \mathrm{SiCH}_{3}, \mathrm{Si}\left(\mathrm{CH}_{3}\right)_{2}\right)$

${ }^{13}$ C-NMR (100 MHz, $\left.\mathrm{CDCl}_{3}\right) 159.6$ (q), 141.8 (q), 134.3 (t), 130.9 (t), 130.8 (q), 129.2 (t), $127.8(\mathrm{t}), 113.7(\mathrm{t}), 78.0(\mathrm{t}), 73.3(\mathrm{t}), 72.5(\mathrm{~s}), 70.2(\mathrm{~s}), 57.0(\mathrm{t}), 55.3(\mathrm{p}), 45.3(\mathrm{t}), 43.3(\mathrm{t}), 40.8$ (s), 40.0 (s), 38.7 (s), 31.8 (s), 31.0 (s), 29.6 (s), 27.6 (s), 26.1 (s), 25.9 (p), 25.9 (p), 25.2 (s), $22.6(\mathrm{~s}), 18.3$ (q), 18.1 (q), 14.0 (p), -4.2 (p), -4.5 (p), -4.6 (p), -4.7 (p) 
IR (Si, Film) $\widetilde{v}_{\max }=2930,2857,1608,1514,1462,1255,1113,835,775$

HRMS $\left(150{ }^{\circ} \mathrm{C} 60 \mathrm{eV}\right): \mathrm{m} / z$ calcd for $\mathrm{C}_{41} \mathrm{H}_{72} \mathrm{O}_{4} \mathrm{Si}_{2}: 684.4969$; found 684.4957

Optical Rotation: $[\alpha]_{\mathrm{D}}^{20}=-1.1(\mathrm{c}=0.3$, acetone $)$

$\boldsymbol{R}_{\mathbf{f}}(10: 1 \mathrm{Hex} / \mathrm{EtOAc})=0.52$

\section{$(S, E)-1-((1 R, 2 R, 3 a S, 6 a S)-2-(t e r t-b u t y l d i m e t h y l s i l y l o x y)-5-(5-(4-)$}

methoxybenzyloxy)pentyl)-1,2,3,3a,6,6a-hexahydropentalen-1-yl)oct-1-en-3-ol, 17b

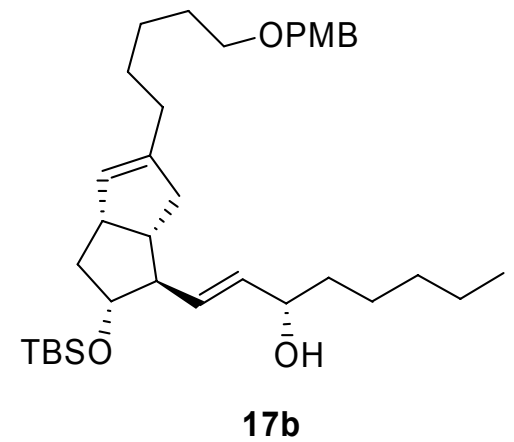

$\mathrm{C}_{35} \mathrm{H}_{58} \mathrm{O}_{4} \mathrm{Si} \quad \mathrm{M}_{\mathrm{r}}=570.92$

${ }^{1}$ H NMR (400 MHz, $\left.\mathrm{CDCl}_{3}\right) 7.26(2 \mathrm{H}, \mathrm{d}, J$ 8.6, $\mathrm{ArH}), 6.88(2 \mathrm{H}, \mathrm{d}, J$ 8.6, $\mathrm{ArH})$, 5.59-5.48 (1H, overlapping dd, $J 15.4,4.3, H \mathrm{C}=\mathrm{CH}), 5.59-5.48(1 \mathrm{H}$, overlapping $\mathrm{dd}, J 15.4,5.3$, $\mathrm{HC}=\mathrm{CH}), 5.24(1 \mathrm{H}, \mathrm{d}, J 1.3$, vinylic in ring $), 4.43\left(2 \mathrm{H}, \mathrm{s}, \mathrm{OCH}_{2} \mathrm{Ar}\right), 4.10-4.03(1 \mathrm{H}, \mathrm{m}$, $\mathrm{HC}=\mathrm{CHCHOH}), 3.80\left(3 \mathrm{H}, \mathrm{s}, \mathrm{ArOCH}_{3}\right), 3.73(1 \mathrm{H}, \mathrm{ddd}, J$ 9.8, 9.5, 6.8, CHOTBS $), 3.43(2 \mathrm{H}$, dd, $J$ 6.8, 6.6, , alkyl $\left.\mathrm{CH}_{2}\right), 2.94\left(1 \mathrm{H}\right.$, dddd, $J$ 8.6, 8.6, 8.5, 1.8, $\left.\mathrm{CHCH}_{2} \mathrm{CHOTBS}\right), 2.40(1 \mathrm{H}$, $\mathrm{dd}, J 16.6,9.0, \mathrm{HC}=\mathrm{CCHH}), 2.25(1 \mathrm{H}, \mathrm{dddd}, J$ 9.5, 9.5, 8.9, 1.8, $H \mathrm{HHCHC}=\mathrm{CH}), 2.18(1 \mathrm{H}$, ddd, $J$ 12.2, 8.8, 7.0, CHHCHOTBS), 2.04-1.93 (4H, m, $H \mathrm{CHC}=\mathrm{CH}, \mathrm{HC}=\mathrm{CCH} H$, alkyl $\left.\mathrm{CH}_{2}\right), 1.61\left(2 \mathrm{H}\right.$, dddd, $J$ 7.3, 7.1, 6.8, 6.8, alkyl $\left.\mathrm{CH}_{2}\right), 1.59-1.57(1 \mathrm{H}, \mathrm{m}, \mathrm{OH}), 1.56-1.24(15 \mathrm{H}$, m, 7 x alkyl $\left.\mathrm{CH}_{2}, \mathrm{CH} H \mathrm{CHOTBS}\right), 0.93-0.84\left(3 \mathrm{H}, \mathrm{m}\right.$, alkyl $\left.\mathrm{CH}_{3}\right), 0.86\left(9 \mathrm{H}, \mathrm{s}, \mathrm{SiC}\left(\mathrm{CH}_{3}\right)_{3}\right)$, $0.02\left(9 \mathrm{H}, \mathrm{s}, \mathrm{Si}\left(\mathrm{CH}_{3}\right)_{2}\right)$

${ }^{13}$ C-NMR (100 MHz, $\left.\mathrm{CDCl}_{3}\right) 159.1$ (q), 141.8 (q), 134.1 (t), 133.1 (t), 130.8 (q), 129.2 (t), $127.8(\mathrm{t}), 113.8(\mathrm{t}), 77.8(\mathrm{t}), 73.1(\mathrm{t}), 72.5(\mathrm{~s}), 70.1(\mathrm{~s}), 57.3(\mathrm{t}), 55.3(\mathrm{p}), 45.4(\mathrm{t}), 43.2(\mathrm{t})$, 
40.7 (s), 39.9 (s), 37.3 (s), 31.8 (s), 31.0 (s), 29.6 (s), 27.6 (s), 26.1 (s), 25.9 (p), 25.3 (p), 22.6 $(\mathrm{s}), 18.1(\mathrm{q}), 14.0(\mathrm{p}),-4.4(\mathrm{p}),-4.6(\mathrm{p})$

IR (Si, Film) $\widetilde{v}_{\max }=3437,2930,2857,1613,1587,1513,1463,1360,1302,1249,1172$

HRMS $\left(140{ }^{\circ} \mathrm{C} 50 \mathrm{eV}\right): \mathrm{m} / z$ calcd for $\mathrm{C}_{35} \mathrm{H}_{58} \mathrm{O}_{4} \mathrm{Si}: 570.4104$; found 570.4394

Optical Rotation: $[\alpha]_{\mathrm{D}}^{20}=-3.3(\mathrm{c}=0.48$, acetone $)$

$\boldsymbol{R}_{\mathbf{f}}(3: 1 \mathrm{Hex} /$ EtOAc $)=0.37$

tert-butyl((2R,3R,3aS,6aS)-3-((R,E)-3-(tert-butyldimethylsilyloxy)-4-m-tolylbut-1-enyl)-5(5-(4-methoxybenzyloxy)pentyl)-1,2,3,3a,4,6a-hexahydropentalen-2yloxy)dimethylsilane, 17d

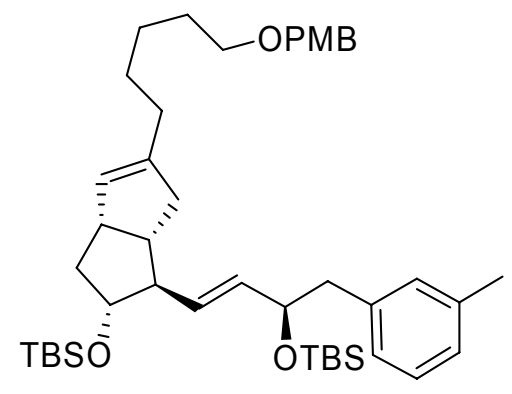

$17 d$

$\mathbf{C}_{44} \mathbf{H}_{70} \mathbf{O}_{4} \mathbf{S i}_{2} \quad \mathbf{M}_{\mathbf{r}}=719.20$

${ }^{1} \mathbf{H}$ NMR (400 MHz, $\left.\mathrm{CDCl}_{3}\right) 7.26$ (2H, d, J 8.6; $\left.\mathrm{ArH}\right), 7.14$ (1H, dd, J 7.6, 7.3, ArH), 7.026.95 (3H, m, ArH), 6.88 (2H, d, $J$ 8.6, ArH), 5.56-5.42 (1H, overlapping dd, $J$ 15.3, 6.3, $H \mathrm{C}=\mathrm{CH}), 5.56-5.42(1 \mathrm{H}$, overlapping dd, $J 15.3,6.9, \mathrm{HC}=\mathrm{CH}), 5.23(1 \mathrm{H}, \mathrm{d}, J 1.3$, vinylic in ring), $4.43\left(2 \mathrm{H}, \mathrm{s}, \mathrm{OCH}_{2} \mathrm{Ar}\right), 4.22(1 \mathrm{H}, \mathrm{ddd}, J$ 7.6, 5.9, 5.4, $\mathrm{HC}=\mathrm{CHCHOTBS}), 3.80(3 \mathrm{H}, \mathrm{s}$, $\left.\mathrm{ArOCH}_{3}\right), 3.72$ (1H, ddd, $J$ 9.6, 9.5, 6.8, CHOTBS), 3.44 (2H, $J$ 6.8, 6.6, alkyl $\mathrm{CH}_{2}$ ), 3.01$2.88\left(1 \mathrm{H}, \mathrm{m}, \mathrm{CHCH}_{2} \mathrm{CHOTBS}\right), 2.77-2.64\left(1 \mathrm{H}\right.$, overlapping dd, $J$ 13.1, 4.5, $\left.\mathrm{HHCArCH}_{3}\right)$,

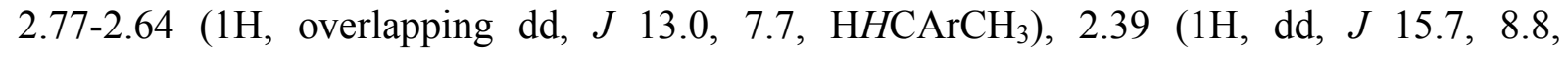
$\mathrm{HC}=\mathrm{CC} H \mathrm{H}), 2.31\left(3 \mathrm{H}, \mathrm{s}, \mathrm{ArCH}_{3}\right), 2.25(1 \mathrm{H}, \mathrm{dddd}, J$ 9.1, 8.8, 8.5, 2.0, $\mathrm{HCHCHC}=\mathrm{CH}), 2.20-$ $2.14(1 \mathrm{H}, \mathrm{m}, \mathrm{CHHCHOTBS}), 2.05-1.91\left(4 \mathrm{H}, \mathrm{m}, \mathrm{HCHC}=\mathrm{CH}, \mathrm{HC}=\mathrm{CCH} H\right.$, alkyl $\left.\mathrm{CH}_{2}\right), 1.61$ (2H, dddd, $J$ 7.3, 7.3, 6.8, 6.6, alkyl $\left.\mathrm{CH}_{2}\right), 1.48-1.29\left(6 \mathrm{H}, \mathrm{m}, 3\right.$ x alkyl $\left.\mathrm{CH}_{2}\right), 1.29-1.22(1 \mathrm{H}$, 
m, CHHCHOTBS), $0.86\left(9 \mathrm{H}, \mathrm{s}, \mathrm{SiC}\left(\mathrm{CH}_{3}\right)_{3}\right), 0.80\left(9 \mathrm{H}, \mathrm{s}, \mathrm{SiC}\left(\mathrm{CH}_{3}\right)_{3}\right), 0.02\left(6 \mathrm{H}, \mathrm{s}, \mathrm{Si}\left(\mathrm{CH}_{3}\right)_{2}\right)$, $-0.14\left(3 \mathrm{H}, \mathrm{s}, \mathrm{SiCH}_{3}\right),-0.22\left(3 \mathrm{H}, \mathrm{s}, \mathrm{SiCH}_{3}\right)$

${ }^{13}$ C-NMR (100 MHz, $\left.\mathrm{CDCl}_{3}\right) 159.1$ (q), 141.9 (q), 139.0 (q), 137.3 (q), 134.4 (t), 131.9 (t), $130.9(\mathrm{t}), 130.8(\mathrm{q}), 129.2(\mathrm{t}), 127.8(\mathrm{t}), 127.8(\mathrm{t}), 126.9(\mathrm{t}), 126.6(\mathrm{t}), 113.8(\mathrm{t}), 77.8(\mathrm{t}), 75.3$ $(\mathrm{t}), 72.5(\mathrm{~s}), 70.2(\mathrm{~s}), 57.3(\mathrm{t}), 55.3(\mathrm{p}), 45.4(\mathrm{t}), 45.3(\mathrm{~s}), 43.2(\mathrm{t}), 40.8(\mathrm{~s}), 40.1(\mathrm{~s}), 31.0(\mathrm{~s})$, 29.6 (s), 27.6 (s), 26.1 (s), 25.9 (p), 25.8 (p), 21.3 (p), 18.2 (q), 18.1 (q), -4.4 (p), -4.6 (p), -4.6 (p), $-5.1(\mathrm{p})$

IR (Si, Film) $\widetilde{v}_{\max }=2929,2856,1714,1610,1513,1462,1360,1249,1112,835$

HRMS $\left(160{ }^{\circ} \mathrm{C} 70 \mathrm{eV}\right): \mathrm{m} / z$ calcd for $\mathrm{C}_{44} \mathrm{H}_{70} \mathrm{O}_{4} \mathrm{Si}_{2}:$ : 718.4813; found 718.4801

Optical Rotation: $[\alpha]_{\mathrm{D}}^{20}=+1.4(\mathrm{c}=0.66$, acetone $)$

$\boldsymbol{R}_{\mathbf{f}}(10: 1 \mathrm{Hex} / \mathrm{EtOAc})=0.39$

\section{$\underline{(R, E)-4-((1 R, 2 R, 3 a S, 6 a S)-2-(t e r t-b u t y l d i m e t h y l s i l y l o x y)-5-(5-(4-~}$}

methoxybenzyloxy)pentyl)-1,2,3,3a,6,6a-hexahydropentalen-1-yl)-1-m-tolylbut-3-en-2-ol, $\underline{17 \mathrm{e}}$

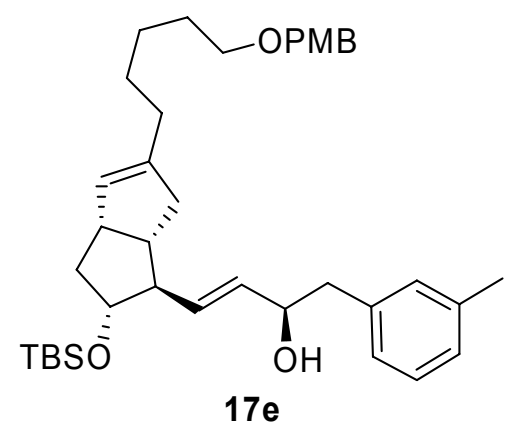

$\mathrm{C}_{38} \mathrm{H}_{56} \mathrm{O}_{4} \mathrm{Si} \quad \mathbf{M}_{\mathbf{r}}=604.93$

${ }^{1}$ H NMR (400 MHz, $\left.\mathrm{CDCl}_{3}\right) 7.26(2 \mathrm{H}, \mathrm{d}, J$ 8.6, $\mathrm{ArH}), 7.18(1 \mathrm{H}, \mathrm{dd}, J$ 7.6, 7.4, $\mathrm{ArH})$, 7.08$7.01(3 \mathrm{H}, \mathrm{m}, \mathrm{Ar} H), 6.88(2 \mathrm{H}, \mathrm{d}, J$ 8.6, $\mathrm{Ar} H), 5.65-5.54(1 \mathrm{H}$, overlapping dd, $J 15.3, H \mathrm{C}=\mathrm{CH})$, 5.65-5.54 (1H, overlapping dd, $J 15.3, \mathrm{HC}=\mathrm{CH}), 5.24(1 \mathrm{H}, \mathrm{d}, J 1.3$, vinylic in ring), $4.43(2 \mathrm{H}$, s, $\left.\mathrm{OCH}_{2} \mathrm{Ar}\right), 4.37-4.30(1 \mathrm{H}, \mathrm{m}, \mathrm{HC}=\mathrm{CHCHOH}), 3.80\left(3 \mathrm{H}, \mathrm{s}, \mathrm{ArOCH}_{3}\right), 3.74(1 \mathrm{H}, \mathrm{ddd}, J$ 9.6, 9.6, 7.1, CHOTBS), $3.44\left(2 \mathrm{H}, \mathrm{dd}, J\right.$ 6.8, 6.8, alkyl $\left.\mathrm{CH}_{2}\right), 2.98-2.90(1 \mathrm{H}, \mathrm{m}$, 
$\left.\mathrm{CHCH}_{2} \mathrm{CHOTBS}\right), 2.85$ (1H, ddd, $J$ 13.3, 13.3, 4.4, $\left.\mathrm{CHOHCHHArCH}_{3}\right), 2.71(1 \mathrm{H}, \mathrm{dd}, J$ 13.3, 8.3, $\left.\mathrm{CHOHCHHArCH}_{3}\right), 2.47-2.36(1 \mathrm{H}, \mathrm{m}, \mathrm{HC}=\mathrm{CCHH}), 2.34 \mathrm{~m}\left(3 \mathrm{H}, \mathrm{s}, \mathrm{ArCH}_{3}\right), 2.29(1 \mathrm{H}$, ddd, $J$ 8.8, 8.8, 8.7, HCHCHC=CH), $2.18(1 \mathrm{H}$, ddd, $J$ 12.3, 8.8, 7.0, CHHCHOTBS $), 2.06-$ $1.93\left(4 \mathrm{H}, \mathrm{m}, \mathrm{HCHC}=\mathrm{CH}, \mathrm{HC}=\mathrm{CCH} H\right.$, alkyl $\left.\mathrm{CH}_{2}\right), 1.61(2 \mathrm{H}$, dddd, $J$ 7.3, 7.1, 6.8, 6.6, alkyl $\left.\mathrm{CH}_{2}\right), 1.47-1.23\left(8 \mathrm{H}, \mathrm{m}, 3 \mathrm{x}\right.$ alkyl $\left.\mathrm{CH}_{2}, \mathrm{CH} H \mathrm{CHOTBS}, \mathrm{OH}\right), 0.86\left(9 \mathrm{H}, \mathrm{s}, \mathrm{SiC}\left(\mathrm{CH}_{3}\right)_{3}\right), 0.02$ $\left(6 \mathrm{H}, \mathrm{s}, \mathrm{Si}\left(\mathrm{CH}_{3}\right)_{2}\right)$

HRMS $\left(150{ }^{\circ} \mathrm{C} 70 \mathrm{eV}\right): \mathrm{m} / z$ calcd for $\mathrm{C}_{38} \mathrm{H}_{56} \mathrm{O}_{4} \mathrm{Si}: 604.3948$; found 604.3957

$\boldsymbol{R}_{\mathbf{f}}(3: 1 \mathrm{Hex} / \mathrm{EtOAc})=0.38$

tert-butyl((2R,3R,3aS,6aS)-5-(5-(4-methoxybenzyloxy)pentyl)-3-((E)-4-m-tolylbut-1-

enyl)-1,2,3,3a,4,6a-hexahydropentalen-2-yloxy)dimethylsilane, $17 \mathrm{f}$

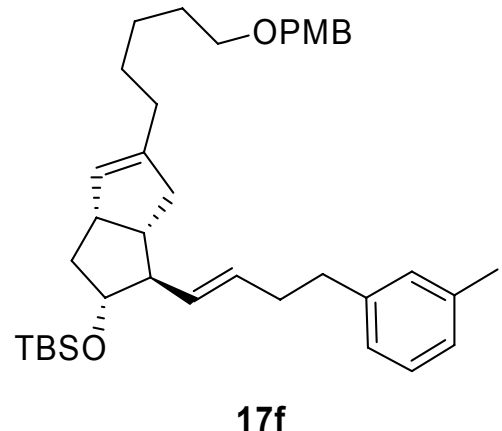

$\mathrm{C}_{38} \mathrm{H}_{56} \mathrm{O}_{3} \mathrm{Si} \quad \mathrm{M}_{\mathrm{r}}=588.93$

${ }^{1} \mathbf{H}$ NMR $\left(400 \mathrm{MHz}, \mathrm{CDCl}_{3}\right) 7.25(2 \mathrm{H}, \mathrm{d}, J$ 8.8, $\mathrm{ArH}), 7.15(1 \mathrm{H}, \mathrm{dd}, J$ 7.8, 7.3, $\mathrm{ArH}), 7.03$ $6.96(3 \mathrm{H}, \mathrm{m}, \mathrm{Ar} H), 6.86(2 \mathrm{H}, \mathrm{d}, J 8.8, \operatorname{Ar} H), 5.51(1 \mathrm{H}, \mathrm{ddd}, J 15.1,6.6,6.6$, vinylic in chain), $5.31(1 \mathrm{H}$, ddd, $J 15.1,8.5,1.3$, vinylic in chain), $5.23(1 \mathrm{H}, \mathrm{dd}, J 3.5,2.0$, vinylic in ring), 4.44 $\left(2 \mathrm{H}, \mathrm{s}, \mathrm{OCH}_{2} \mathrm{Ar}\right), 3.81\left(3 \mathrm{H}, \mathrm{s}, \mathrm{OCH}_{3}\right), 3.70$ (1H, ddd, $J$ 9.6, 9.6, 7.0, CHOTBS), 3.44 (2H, t, $J$ 6.6, alkyl $\left.\mathrm{CH}_{2}\right), 2.92\left(1 \mathrm{H}, \mathrm{dd}, J\right.$ 8.8, 8.1, $\left.\mathrm{CHCH}_{2} \mathrm{CHOTBS}\right), 2.72-2.58\left(2 \mathrm{H}, \mathrm{m}\right.$, benzylic $\left.\mathrm{CH}_{2}\right)$, $2.39(1 \mathrm{H}, \mathrm{dd}, J 16.2,8.6, \mathrm{HC}=\mathrm{CCHH}), 2.36-2.28\left(2 \mathrm{H}, \mathrm{m}, \mathrm{HC}=\mathrm{CHCH}_{2}\right), 2.33\left(3 \mathrm{H}, \mathrm{s}, \mathrm{CH}_{3}\right)$, 2.21 (1H, ddd, $J$ 9.7, 8.8, 8.6, CHCHCHOTBS), 2.17 (1H, ddd, $J$ 12.3, 8.8, 7.0, CHHCHOTBS), 2.05-1.96 (3H, m, $\mathrm{HC}=\mathrm{CCH} H$, alkyl $\left.\mathrm{CH}_{2}\right), 1.91(1 \mathrm{H}$, ddd, $J$ 9.7, 9.6, 8.5, CHCHOTBS), 1.62 (2H, dddd, $J$ 7.1, 7.1, 6.8, 6.8, alkyl $\left.\mathrm{CH}_{2}\right), 1.48-1.31(4 \mathrm{H}, \mathrm{m}, 2 \mathrm{x}$ alkyl $\left.\mathrm{CH}_{2}\right), 1.26(1 \mathrm{H}, \mathrm{ddd}, J 12.3,9.6,8.1, \mathrm{CH} H \mathrm{CHOTBS}), 0.87\left(9 \mathrm{H}, \mathrm{s}, \mathrm{SiC}\left(\mathrm{CH}_{3}\right)_{3}\right), 0.02(6 \mathrm{H}, \mathrm{s}$, $\left.\mathrm{Si}\left(\mathrm{CH}_{3}\right)_{2}\right)$ 
${ }^{13}$ C-NMR (100 MHz, $\mathrm{CDCl}_{3}$ ) 159.3 (q), 142.4 (q), 142.0 (q), 137.9 (q), 132.7 (t), 131.0 (q), $131.0(\mathrm{t}), 129.4(\mathrm{t}), 129.3(\mathrm{t}), 128.3(\mathrm{t}), 128.0(\mathrm{t}), 126.6(\mathrm{t}), 125.6(\mathrm{t}), 113.9(\mathrm{t}), 78.1(\mathrm{t}), 72.7$ (s), $70.3(\mathrm{~s}), 58.0(\mathrm{t}), 55.4(\mathrm{t}), 45.4(\mathrm{t}), 43.6(\mathrm{t}), 40.8(\mathrm{~s}), 40.1(\mathrm{~s}), 36.2(\mathrm{~s}), 35.0(\mathrm{~s}), 31.2(\mathrm{~s})$, 29.8 (s), 27.8 (s), 26.2 (s), 26.0 (p), 21.6 (p), 18.4 (q), -4.4 (p), -4.4 (p)

IR $\left(\mathrm{Si}\right.$, Film) $\tilde{v}_{\max }=2928,2855,1612,1513,1172,1113,1039,1005,908,836,700$ HRMS $\left(160{ }^{\circ} \mathrm{C} 70 \mathrm{eV}\right): \mathrm{m} / z$ calcd for $\mathrm{C}_{38} \mathrm{H}_{56} \mathrm{O}_{3} \mathrm{Si}: 588.3999$; found 588.3984

Optical Rotation: $[\alpha]_{\mathrm{D}}^{20}=+0.5\left(\mathrm{c}=1.05, \mathrm{CHCl}_{3}\right)$

$\boldsymbol{R}_{\mathbf{f}}(3: 1 \mathrm{Hex} /$ EtOAc $)=0.48$ 


\section{Derivatives}

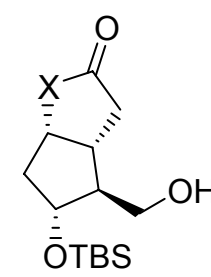

1. Swern Ox.

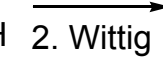
$86-92 \%$

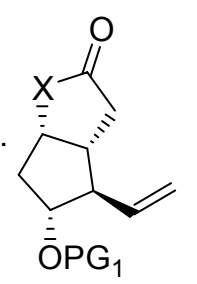

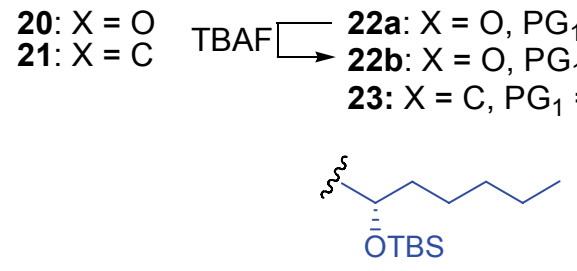

9
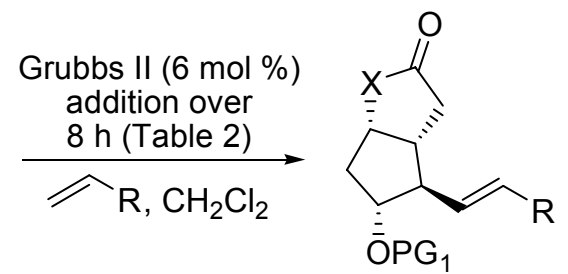

24a-b: $X=O, P_{1}=$ TBS

24c: $X=O, P G_{1}=H$

25: $X=C, P G_{1}=T B S$

$\underline{\text { Table } 2 \text { Prostaglandin/Carbacyclin Cross Metathesis (Yields Not Optimized) }}{ }^{\mathrm{a}}$

\begin{tabular}{|c|c|c|c|c|c|c|}
\hline entry & $\begin{array}{c}\text { bicyclic } \\
\text { olefin }\end{array}$ & $\mathrm{PG}_{1}$ & $\begin{array}{l}\omega \text {-side chain } \\
\text { olefin } \mathrm{R}^{\mathrm{b}}\end{array}$ & product & $E: Z$ & $\begin{array}{l}\text { yield } \\
(\%)^{\mathrm{c}}\end{array}$ \\
\hline 1 & $22 a$ & TBS & & $24 a$ & $6: 1$ & 76 \\
\hline $2^{\mathrm{d}}$ & $22 a$ & TBS & & $24 a$ & $12: 1$ & 68 \\
\hline $3^{\mathrm{e}}$ & $22 a$ & TBS & & $24 a$ & $17: 1$ & 84 \\
\hline 4 & $22 a$ & TBS & & $24 b$ & $12: 1$ & $60^{\mathrm{f}}$ \\
\hline 5 & $22 b$ & $\mathrm{H}$ & & $24 c$ & $14: 1$ & 70 \\
\hline 6 & 23 & TBS & & 25 & $7: 1$ & 69 \\
\hline $7^{\mathrm{g}}$ & 23 & TBS & & 25 & $10: 1$ & 65 \\
\hline $8^{\mathrm{e}}$ & 23 & TBS & ŌTBS & 25 & $10: 1$ & 86 \\
\hline
\end{tabular}

Isolated yields. ${ }^{\mathrm{d}}$ Product described in entry 1 was re-exposed to Grubbs' II for $12 \mathrm{~h}$ at $40{ }^{\circ} \mathrm{C}$.

${ }^{\text {e }}$ Catalyst added over $12 \mathrm{hrs}$ at $40{ }^{\circ} \mathrm{C} .{ }^{\mathrm{f}} 30 \%$ homodimerized $\omega$-side chain was recovered. ${ }^{\mathrm{g}}$ Product described in entry 6 was re-exposed to Grubbs' II for $12 \mathrm{~h}$ at $40{ }^{\circ} \mathrm{C}$.

A representative procedure for the cross metathesis reaction is as previously described (vide supra). 


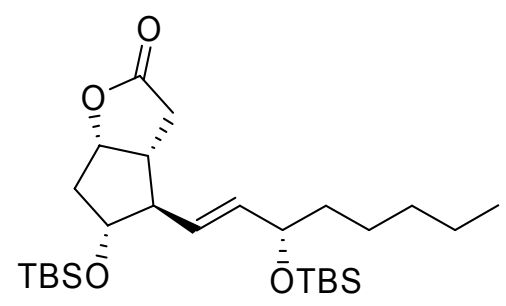

24a

$\mathbf{C}_{27} \mathbf{H}_{52} \mathbf{O}_{4} \mathbf{S i}_{2} \quad \mathbf{M}_{\mathbf{r}}=496.87$

${ }^{1} \mathbf{H}$ NMR $\left(600 \mathrm{MHz}, \mathrm{CDCl}_{3}\right) 5.50(1 \mathrm{H}, \mathrm{ddd}, J 15.4,5.8,1.0, \mathrm{HC}=\mathrm{CH}), 5.37(1 \mathrm{H}, \mathrm{ddd}, J$ 15.4, 7.7, 1.0, $\mathrm{HC}=\mathrm{CH}), 4.94(1 \mathrm{H}, \mathrm{ddd}, J 7.1,7.1,2.1, H \mathrm{COC}=\mathrm{O}), 4.04(1 \mathrm{H}$, ddd, $J 5.9,5.7,5.7$, $\mathrm{C}=\mathrm{CHCHOTBS}$ ), 3.98 (1H, ddd, $J$ 5.3, 5.3, 5.3, CHOTBS), 2.75 (1H, dd, $J$ 18.1, 10.4, $\mathrm{OC}=\mathrm{OCHH}), 2.67-2.61(1 \mathrm{H}, \mathrm{m}, \mathrm{HC}=\mathrm{CHCHCH}), 2.49(1 \mathrm{H}, \mathrm{dd}, J 18.1,2.8, \mathrm{OC}=\mathrm{OCH} H), 2.44$ (dd, J 12.9, 6.0, $\left.\mathrm{H}_{2} \mathrm{C}=\mathrm{CHCH}\right), 2.24$ (1H, ddd, J 14.8, 7.0, 5.8, CHHCHOTBS), 1.98 (1H, ddd, $J$ 14.8, 4.9, 1.9, СНHCHOTBS), 1.51-1.44 (1H, m, СНОТВSСHН), 1.44-1.37 (1H, m, CHOTBSCHH), 1.34-1.20 (6H, m, 3 x alkyl $\left.\mathrm{CH}_{2}\right), 0.90-0.88\left(3 \mathrm{H}, \mathrm{m}\right.$, alkyl $\left.\mathrm{CH}_{3}\right), 0.88(9 \mathrm{H}, \mathrm{s}$, $\left.\mathrm{SiC}\left(\mathrm{CH}_{3}\right)_{3}\right), 0.87\left(9 \mathrm{H}, \mathrm{s}, \mathrm{SiC}\left(\mathrm{CH}_{3}\right)_{3}\right), 0.05\left(3 \mathrm{H}, \mathrm{s}, \mathrm{SiCH}_{3}\right), 0.04\left(3 \mathrm{H}, \mathrm{s}, \mathrm{SiCH}_{3}\right), 0.03(3 \mathrm{H}, \mathrm{s}$, $\left.\mathrm{SiCH}_{3}\right), 0.00\left(3 \mathrm{H}, \mathrm{s}, \mathrm{SiCH}_{3}\right)$

${ }^{13}$ C-NMR (150 MHz, $\left.\mathrm{CDCl}_{3}\right) 177.0(\mathrm{q}), 135.8(\mathrm{t}), 128.4(\mathrm{t}), 83.4(\mathrm{t}), 79.0(\mathrm{t}), 72.9(\mathrm{t}), 56.1$ $(\mathrm{t}), 42.3(\mathrm{t}), 40.7(\mathrm{~s}), 38.4(\mathrm{~s}), 35.0(\mathrm{~s}), 31.8(\mathrm{~s}), 25.9$ (p), 25.7 (p), 25.0 (s), 22.6 (s), 18.2 (q), $18.0(\mathrm{q}), 14.0(\mathrm{p}),-4.3(\mathrm{p}),-4.7(\mathrm{p}),-4.8(\mathrm{p}),-4.9(\mathrm{p})$

IR (Si, Film) $\widetilde{v}_{\max }=2930,2857,2362,1752,1472,1249,1165,1119,974$

HRMS $\left(160{ }^{\circ} \mathrm{C} 70 \mathrm{eV}\right): \mathrm{m} / z$ calcd for $\mathrm{C}_{27} \mathrm{H}_{52} \mathrm{O}_{4} \mathrm{Si}_{2}: 496.3404$; found 496.3395

Optical Rotation: $[\alpha]_{\mathrm{D}}^{20}=-19.2(\mathrm{c}=0.43$, acetone $)$

$\boldsymbol{R}_{\mathbf{f}}(1: 1 \mathrm{Hex} / \mathrm{EtOAc})=0.64$ 


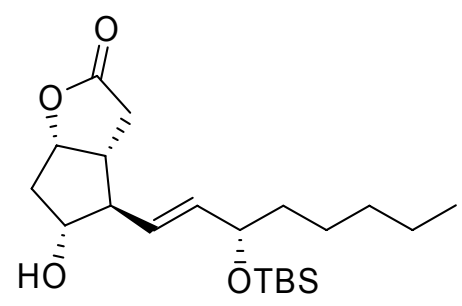

24c

$\mathrm{C}_{21} \mathrm{H}_{38} \mathrm{O}_{4} \mathrm{Si} \quad \mathrm{M}_{\mathrm{r}}=382.61$

${ }^{1}$ H NMR $\left(400 \mathrm{MHz}, \mathrm{CDCl}_{3}\right) 5.60(1 \mathrm{H}, \mathrm{dd}, J$ 15.3, 5.7, $\mathrm{HC}=\mathrm{CH}), 5.41(1 \mathrm{H}, \mathrm{dd}, J$ 15.3, 9.0, $\mathrm{HC}=\mathrm{CH}), 4.97-4.86(1 \mathrm{H}, \mathrm{m}, \mathrm{HCOC}=\mathrm{O}), 4.13-3.95(2 \mathrm{H}, \mathrm{m}, \mathrm{CHOH}, \mathrm{CHOTBS}), 2.85-2.30$ $\left(5 \mathrm{H}, \mathrm{m}, \mathrm{OC}=\mathrm{OCH}_{2} \mathrm{CH}, \mathrm{HCHC}=\mathrm{CH}, \mathrm{CH}_{2} \mathrm{CHOH}, \mathrm{OC}=\mathrm{OCH} H\right), 2.01(1 \mathrm{H}, \mathrm{ddd}, J 15.0,6.9,2.8$, $\mathrm{OC}=\mathrm{OCHH}), 1.55-1.41\left(2 \mathrm{H}, \mathrm{m}\right.$, alkyl $\left.\mathrm{CH}_{2}\right)$ 1.39-1.21 $\left(6 \mathrm{H}, \mathrm{m}, 3 \mathrm{x}\right.$ alkyl $\left.\mathrm{CH}_{2}\right), 0.89(9 \mathrm{H}, \mathrm{s}$, $\left.\mathrm{SiC}\left(\mathrm{CH}_{3}\right)_{3}\right), 0.92-0.85\left(3 \mathrm{H}, \mathrm{m}\right.$, alkyl $\left.\mathrm{CH}_{3}\right), 0.05\left(3 \mathrm{H}, \mathrm{s}, \mathrm{SiCH}_{3}\right), 0.03\left(3 \mathrm{H}, \mathrm{s}, \mathrm{SiCH}_{3}\right)$

${ }^{13}$ C-NMR (100 MHz, $\left.\mathrm{CDCl}_{3}\right) 176.6$ (q), $137.6(\mathrm{t}), 127.5$ (t), 82.6 (t), 76.8 (t), $72.3(\mathrm{t}), 56.1$ (t), $42.6(\mathrm{t}), 39.8(\mathrm{~s}), 38.2(\mathrm{~s}), 34.3(\mathrm{~s}), 31.6(\mathrm{~s}), 24.8(\mathrm{~s}), 22.5(\mathrm{~s}), 25.8(\mathrm{p}), 18.1(\mathrm{q}), 13.9$ (p), $4.4(\mathrm{p}),-4.8(\mathrm{p})$

IR (Si, Film) $\widetilde{v}_{\max }=3396,2925,2840,1761,1168$

HRMS $\left(150{ }^{\circ} \mathrm{C} 70 \mathrm{eV}\right): \mathrm{m} / z$ calcd for $\mathrm{C}_{21} \mathrm{H}_{38} \mathrm{O}_{4} \mathrm{Si}: 382.2539$; found 382.2547

Optical Rotation: $[\alpha]_{\mathrm{D}}^{20}=-14.7\left(\mathrm{c}=0.48, \mathrm{CH}_{2} \mathrm{Cl}_{2}\right)$

$\boldsymbol{R}_{\mathbf{f}}(1: 1 \mathrm{Hex} / \mathrm{EtOAc})=0.35$ 


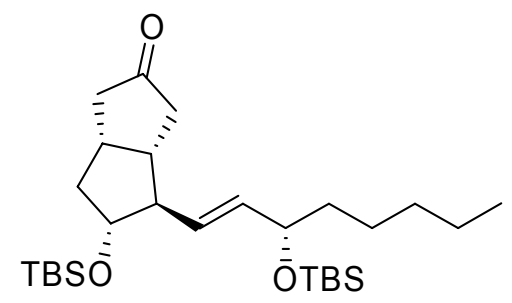

25

$\mathbf{C}_{28} \mathbf{H}_{54} \mathbf{O}_{3} \mathbf{S i}_{2} \quad \mathbf{M}_{\mathbf{r}}=494.90$

${ }^{1} \mathbf{H}$ NMR $\left(600 \mathrm{MHz}, \mathrm{CDCl}_{3}\right)$ 5.51-5.41 (1H, overlapping dd, $\left.J 15.5,5.0, H \mathrm{C}=\mathrm{CH}\right), 5.51-5.41$ $(1 \mathrm{H}$, overlapping dd, $J 15.5,7.2, \mathrm{HC}=\mathrm{CH}), 4.05(1 \mathrm{H}, \mathrm{ddd}, J 6.1,5.9,5.5, \mathrm{HC}=\mathrm{CHCHOTBS})$, 3.95 (1H, ddd, $J$ 6.8, 6.8, 6.8, CHOTBS), 2.75-2.67 (1H, m, $\left.\mathrm{CHCH}_{2} \mathrm{CHOTBS}\right), 2.56(1 \mathrm{H}$, ddd, $J$ 18.7, 10.5, 1.3, $\mathrm{O}=\mathrm{CC} H \mathrm{HCHCH}), 2.47-2.37(1 \mathrm{H}$, overlapping dd, $J$ 9.3, 2.7, $\left.\mathrm{O}=\mathrm{CHHCHCH}_{2}\right), 2.47-2.37(1 \mathrm{H}$, overlapping dd, $J$ 9.6, 1.3, $H \mathrm{CHCHC}=\mathrm{CH}), 2.33(1 \mathrm{H}, \mathrm{ddd}, J$ 13.4, 8.6, 6.8 CHHCHOTBS), 2.25-2.20 (1H, m, O=CHHCHCH$\left.{ }_{2}\right), 2.20-2.14(2 \mathrm{H}, \mathrm{m}$, CHCHOTBS, $\mathrm{O}=\mathrm{CCH} H \mathrm{CHCH}), 1.51-1.44(2 \mathrm{H}, \mathrm{m}, \mathrm{CH} H \mathrm{CHOTBS}, \mathrm{HC}=\mathrm{CHCHOTBSCH} \mathrm{H})$, 1.44-1.37 (1H, m, HC=CHCHOTBSCH $H), 1.36-1.21\left(6 \mathrm{H}, \mathrm{m}, 3 \mathrm{x}\right.$ alkyl $\left.\mathrm{CH}_{2}\right), 0.90-0.87(3 \mathrm{H}$, m, alkyl $\left.\mathrm{CH}_{3}\right), 0.87\left(9 \mathrm{H}, \mathrm{s}, \mathrm{SiC}\left(\mathrm{CH}_{3}\right)_{3}\right), 0.86\left(9 \mathrm{H}, \mathrm{s}, \mathrm{SiC}\left(\mathrm{CH}_{3}\right)_{3}\right), 0.04\left(3 \mathrm{H}, \mathrm{s}, \mathrm{SiCH}_{3}\right), 0.03$ $\left(3 \mathrm{H}, \mathrm{s}, \mathrm{SiCH}_{3}\right), 0.03\left(3 \mathrm{H}, \mathrm{s}, \mathrm{SiCH}_{3}\right), 0.01\left(3 \mathrm{H}, \mathrm{s}, \mathrm{SiCH}_{3}\right)$

${ }^{13}$ C-NMR (100 MHz, $\left.\mathrm{CDCl}_{3}\right) 220.3$ (q), $135.1(\mathrm{t}), 129.7(\mathrm{t}), 79.3(\mathrm{t}), 73.0(\mathrm{t}), 57.5(\mathrm{t}), 46.0$ $(\mathrm{s}), 43.2(\mathrm{~s}), 43.0(\mathrm{t}), 42.4(\mathrm{~s}), 38.5(\mathrm{~s}), 31.8(\mathrm{~s}), 25.9(\mathrm{p}), 25.8(\mathrm{p}), 25.1$ (s), $22.6(\mathrm{~s}), 18.2(\mathrm{q})$, $18.0(\mathrm{q}), 14.0(\mathrm{p}),-4.2(\mathrm{p}),-4.6(\mathrm{p}),-4.7(\mathrm{p}),-4.7(\mathrm{p})$

IR (Si, Film) $\tilde{v}_{\max }=2956,2930,2857,1742,1472,1361,1256,1121,1005,837,776$

HRMS $\left(160{ }^{\circ} \mathrm{C} 70 \mathrm{eV}\right): \mathrm{m} / z$ calcd for $\mathrm{C}_{28} \mathrm{H}_{54} \mathrm{O}_{3} \mathrm{Si}_{2}$ : 494.3611 ; found 494.3601

Optical Rotation: $[\alpha]_{\mathrm{D}}^{20}=-14.2(\mathrm{c}=0.72$, acetone $)$

$\boldsymbol{R}_{\mathbf{f}}(5: 1 \mathrm{Hex} / \mathrm{EtOAc})=0.48$ 


\section{$\underline{5-((3 a S, 5 R, 6 R, 6 a S)-5-h y d r o x y-6-((S, E)-3-h y d r o x y o c t-1-e n y l)-1,3 a, 4,5,6,6 a-}$}

\section{hexahydropentalen-2-yl)pentanoic acid (Isocarbacyclin), 1}

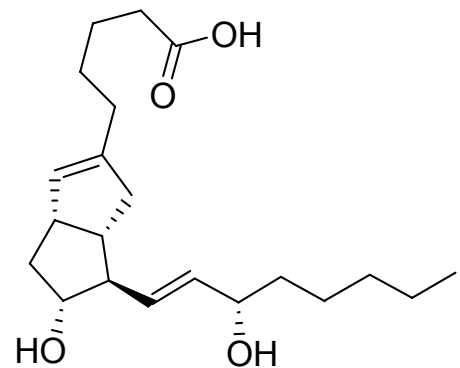

Isocarbacyclin (1)

$\mathrm{C}_{21} \mathrm{H}_{34} \mathrm{O}_{4} \quad \mathbf{M}_{\mathrm{r}}=350.25$

${ }^{1} \mathbf{H}$ NMR $\left(600 \mathrm{MHz}, \mathrm{CDCl}_{3}\right) 5.56(1 \mathrm{H}, \mathrm{dd}, J 15.3,7.2$, vinylic in chain), $5.50(1 \mathrm{H}, \mathrm{dd}, J 15.3$, 8.4, vinylic in chain), $5.29(1 \mathrm{H}, \mathrm{d}, J 1.4$, vinylic in ring), 5.23-3.26 (1H, broad s, $\mathrm{COOH}), 4.06$ $(1 \mathrm{H}, \mathrm{ddd}, J 6.9,6.7,6.7, \mathrm{HC}=\mathrm{CHC}(\mathrm{OH}) H), 3.76(1 \mathrm{H}, \mathrm{ddd}, J 9.6,7.2,7.1, H \mathrm{COH}), 3.00(1 \mathrm{H}$, ddd, $J$ 8.8, 8.6, 2.1, $\left.\mathrm{CHCH}_{2} \mathrm{CHOH}\right), 2.42-2.25\left(5 \mathrm{H}, \mathrm{m}\right.$, alkyl $\mathrm{CH}_{2}, \mathrm{CHCHCHOH}$, $\mathrm{CH} H \mathrm{HCHCH}, \mathrm{CH} H C H O H), 2.06\left(2 \mathrm{H}, \mathrm{dd}, J\right.$ 7.2, 6.9, alkyl $\left.\mathrm{CH}_{2}\right), 1.99(1 \mathrm{H}, \mathrm{bd}, J$ 15.7, $\mathrm{CH} H \mathrm{CHCH}), 1.92(1 \mathrm{H}, \mathrm{ddd}, J$ 9.5, 9.2, 8.8, $\mathrm{CHCHOH}), 1.70-1.54\left(3 \mathrm{H}, \mathrm{m}\right.$, alkyl $\mathrm{CH}_{2}$, $\mathrm{HC}=\mathrm{CHC}(\mathrm{OH}) \mathrm{HCH}), 1.52-1.44\left(3 \mathrm{H}, \mathrm{m}\right.$, alkyl $\left.\mathrm{CH}_{2}, \mathrm{HC}=\mathrm{CHC}(\mathrm{OH}) \mathrm{HCH} H\right), 1.40-1.23(7 \mathrm{H}$, m, 3 x alkyl $\left.\mathrm{CH}_{2}, \mathrm{CH} H \mathrm{CHOH}\right), 0.89\left(3 \mathrm{H}, \mathrm{t}, J 7.0, \mathrm{CH}_{3}\right)$

${ }^{13}$ C-NMR (100 MHz, $\left.\mathrm{CDCl}_{3}\right) 178.1$ (q), 141.1 (q), $135.6(\mathrm{t}), 133.0$ (t), 128.9 (t), $77.3(\mathrm{t}), 73.3$ $(\mathrm{t}), 58.0(\mathrm{t}), 45.6(\mathrm{t}), 44.3(\mathrm{t}), 39.5(\mathrm{~s}), 39.2(\mathrm{~s}), 37.0(\mathrm{~s}), 33.7(\mathrm{~s}), 31.7(\mathrm{~s}), 30.3(\mathrm{~s}), 26.9(\mathrm{~s})$, $25.2(\mathrm{~s}), 24.3(\mathrm{~s}), 22.6(\mathrm{~s}), 14.0(\mathrm{p})$

IR (Si, Film) $\tilde{v}_{\max }=3369,2927,2857,1707,1653,1560,1540,1507,1457,1261,1088,970$

HRMS $\left(170{ }^{\circ} \mathrm{C} 70 \mathrm{eV}\right): \mathrm{m} / z$ calcd for $\mathrm{C}_{21} \mathrm{H}_{34} \mathrm{O}_{4}: 350.2457$; found 350.2432

Optical Rotation: $[\alpha]_{\mathrm{D}}^{20}=+11.8\left(\mathrm{c}=0.46, \mathrm{CH}_{2} \mathrm{Cl}_{2}\right)$

$\boldsymbol{R}_{\mathbf{f}}(\mathrm{EtOAc} / 1 \% \mathrm{AcOH})=0.33$

${ }^{1} \mathrm{H},{ }^{13} \mathrm{C}$ NMR and optical rotation were concurrent with literature values for Isocarbacyclin. See T. Ishikawa; H. Ishii; K. Shimizu; H. Nakao; J. Urano; T. Kudo; S. Saito, J. Org. Chem. 2004, 69, 8133. 


\section{hexahydropentalen-2-yl)pentanoic acid (15R-TIC), 2}

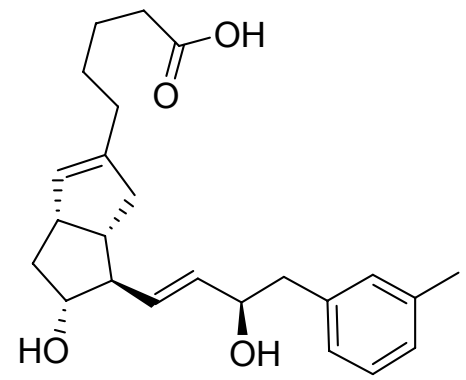

$15 R$-TIC (2)

$\mathbf{C}_{24} \mathbf{H}_{32} \mathbf{O}_{4} \quad \mathbf{M}_{\mathbf{r}}=384.23$

${ }^{1} \mathbf{H}$ NMR $\left(600 \mathrm{MHz}, \mathrm{CDCl}_{3}\right) 7.20(1 \mathrm{H}, \mathrm{dd}, J$ 7.6, 7.5, $\mathrm{ArH}), 7.05(1 \mathrm{H}, \mathrm{d}, J$ 7.6, $\mathrm{ArH}), 7.02$ $(1 \mathrm{H}, \mathrm{s}, \operatorname{Ar} H), 7.00(1 \mathrm{H}, \mathrm{d}, J 7.5, \operatorname{Ar} H), 5.61(1 \mathrm{H}, \mathrm{ddd}, J 15.4,6.7,0.7$, vinylic in chain), 5.44 $(1 \mathrm{H}$, ddd, $J 15.4,8.7,0.7$, vinylic in chain), $5.28(1 \mathrm{H}, \mathrm{d}, J 3.3$, vinylic in ring $), 4.35(1 \mathrm{H}$, ddd, $J$ 6.6, 6.5, 6.4, $\mathrm{HC}=\mathrm{CHC}(\mathrm{OH}) H), 3.63(1 \mathrm{H}, \mathrm{ddd}, J 9.5,7.0,6.9, H \mathrm{COH}), 2.98(1 \mathrm{H}, \mathrm{ddd}, J 9.0$, 8.9, 8.9, $\left.\mathrm{CHCH}_{2} \mathrm{CHOH}\right), 2.86(1 \mathrm{H}, \mathrm{dd}, J$ 13.3, 7.2, CHHAr), 2.79 (1H, dd, $J$ 13.3, 6.3, $\mathrm{CH} H \mathrm{Ar}), 2.42-2.34\left(2 \mathrm{H}, \mathrm{m}, \mathrm{CH}_{2} \mathrm{COOH}\right), 2.33\left(3 \mathrm{H}, \mathrm{s}, \mathrm{CH}_{3}\right), 2.31-2.27(2 \mathrm{H}, \mathrm{m}, \mathrm{CHCHCHOH}$, $\mathrm{CHHCHCH}), 2.26$ (1H, ddd, $J$ 12.5, 8.8, 7.1, $\mathrm{CHHCHOH}), 2.08-1.94\left(3 \mathrm{H}, \mathrm{m}\right.$, alkyl $\mathrm{CH}_{2}$, $\mathrm{CH} H \mathrm{CHCH}), 1.89$ (1H, ddd, $J$ 9.4, 9.3, 9.2, $\mathrm{CHCHOH}), 1.70-1.58$ (2H, m, alkyl $\left.\mathrm{CH}_{2}\right), 1.54-$ $1.45\left(2 \mathrm{H}, \mathrm{m}\right.$, alkyl $\left.\mathrm{CH}_{2}\right), 1.39-1.18(3 \mathrm{H}, \mathrm{CH} H \mathrm{CHOH}, \mathrm{HC}=\mathrm{CHCOH})$

${ }^{13}$ C-NMR (100 MHz, $\left.\mathrm{CDCl}_{3}\right) 178.3$ (q), 141.3 (q), 138.2 (q), 137.8 (q), 134.3 (t), 132.9 (t), $130.4(\mathrm{t}), 128.5(\mathrm{t}), 128.3(\mathrm{t}), 127.4(\mathrm{t}), 126.6(\mathrm{t}), 76.9(\mathrm{~s}), 73.7(\mathrm{t}), 58.1(\mathrm{t}), 45.6(\mathrm{t}), 44.3(\mathrm{t})$, $44.3(\mathrm{t}), 39.5$ (s), 39.3 (s), 33.5 (s), 30.5 (s), 27.0 (s), 24.4 (s), 21.4 (p)

IR (Si, Film) $\tilde{v}_{\max }=3401,2917,2849,1701,1653,1560,1540,1507,1457,1261,1092,1020$

HRMS $\left(170{ }^{\circ} \mathrm{C} 70 \mathrm{eV}\right): \mathrm{m} / z$ calcd for $\mathrm{C}_{24} \mathrm{H}_{32} \mathrm{O}_{4}: 384.2301$; found 384.2287

$\boldsymbol{R}_{\mathbf{f}}(\mathrm{EtOAc} / 1 \% \mathrm{AcOH})=0.52$

${ }^{1} \mathrm{H},{ }^{13} \mathrm{C}$ NMR and optical rotation were concurrent with literature values for $15 R$-TIC. See M. Suzuki; K. Kato; R. Noyori; Y. Watanabe; H. Takechi,; K. Matsumura; B. Långström; Y. Watanabe, Angew. Chem. Int. Ed. Engl. 1996, 35, 334. 
$\underline{\text { enoic acid }\left(\mathrm{PGF}_{2 \alpha}\right), 4}$

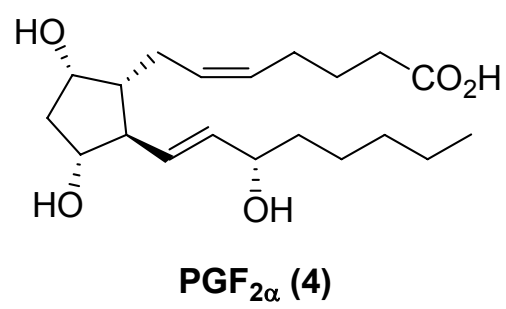

$\mathrm{C}_{20} \mathrm{H}_{34} \mathrm{O}_{5} \quad \mathrm{M}_{\mathrm{r}}=354.24$

${ }^{1} \mathbf{H}$ NMR $\left(600 \mathrm{MHz}, \mathrm{CDCl}_{3}\right)$ 5.88-4.45 (1H, bs, $\left.\mathrm{COOH}\right), 5.58(1 \mathrm{H}, \mathrm{dd}, J 15.3,6.4$, vinylic in chain), 5.51 (1H, dd, $J 15.3,8.6$, vinylic in chain), 5.50-5.44 (1H, m, vinylic in chain), 5.41$5.33(1 \mathrm{H}, \mathrm{m}$, vinylic in chain), $4.19(1 \mathrm{H}, \mathrm{t}, J 4.4, \mathrm{CHOH}), 4.11(1 \mathrm{H}, \mathrm{q}, J 6.6, \mathrm{HC}=\mathrm{CHCHOH})$, 3.98-3.94 $(1 \mathrm{H}, \mathrm{m}, \mathrm{CHOH}), 2.34\left(2 \mathrm{H}, \mathrm{dd}, J\right.$ 7.1, 6.7, alkyl $\left.\mathrm{CH}_{2}\right), 2.34-2.30(1 \mathrm{H}, \mathrm{m}$, $\mathrm{CHCHOH}), 2.26-2.10\left(5 \mathrm{H}, \mathrm{m} \mathrm{CHHCHOH}, 2 \mathrm{x}\right.$ alkyl $\left.\mathrm{CH}_{2}\right), 1.77(1 \mathrm{H}, \mathrm{dd}, J$ 15.1, 2.7, $\mathrm{CH} H \mathrm{CHOH}), 1.74-1.62\left(2 \mathrm{H}, \mathrm{m}\right.$, alkyl $\left.\mathrm{CH}_{2}\right), 1.62-1.54(1 \mathrm{H}, \mathrm{m}, \mathrm{HC}=\mathrm{CHC}(\mathrm{OH}) \mathrm{HCHH}), 1.53-$ $1.44\left(2 \mathrm{H}, \mathrm{m}, \mathrm{CHCH} \mathrm{CH}_{2} \mathrm{CH}, \mathrm{HC}=\mathrm{CHC}(\mathrm{OH}) \mathrm{HCHH}\right), 1.43-1.29\left(6 \mathrm{H}, 3 \mathrm{x}\right.$ alkyl $\left.\mathrm{CH}_{2}\right), 0.88$ $\left(3 \mathrm{H}, \mathrm{t}, \mathrm{J} 6.9, \mathrm{CH}_{3}\right)$

${ }^{13}$ C-NMR (100 MHz, $\left.\mathrm{CDCl}_{3}\right) 177.6(\mathrm{q}), 134.6(\mathrm{t}), 132.7(\mathrm{t}), 129.6(\mathrm{t}), 129.1(\mathrm{t}), 77.9(\mathrm{t}), 73.0$ $(\mathrm{t}), 72.8(\mathrm{t}), 55.6(\mathrm{t}), 50.6(\mathrm{t}), 42.8(\mathrm{~s}), 37.0(\mathrm{~s}), 32.9(\mathrm{~s}), 31.7(\mathrm{~s}), 26.3(\mathrm{~s}), 25.3(\mathrm{~s}), 25.2(\mathrm{~s})$, $24.5(\mathrm{~s}), 22.6(\mathrm{~s}), 14.0(\mathrm{p})$

IR $\left(\mathrm{Si}\right.$, Film) $\widetilde{v}_{\max }=3369,2927,2857,1706,1558,1456,1261,1020,971,875,870,860$

HRMS $\left(150{ }^{\circ} \mathrm{C} 70 \mathrm{eV}\right): \mathrm{m} / z$ calcd for $\mathrm{C}_{20} \mathrm{H}_{34} \mathrm{O}_{5}: 350.2457$; found 350.2444

Optical Rotation: $[\alpha]_{\mathrm{D}}^{20}=+24.9(\mathrm{c}=0.57, \mathrm{THF})$

$\boldsymbol{R}_{\mathbf{f}}($ EtOAc $)=0.18$

${ }^{1} \mathrm{H},{ }^{13} \mathrm{C}$ NMR and optical rotation were concurrent with literature values for $\mathrm{PGF}_{2 \alpha}$. See (a) Y. Sato; M. Takimoto; M. Mori, Chem. Pharm. Bull., 2000, 48, 1753. (b) E. J. Corey; N. M. Weinshenker; T. K. Schaaf; W. Huber, J. Am. Chem. Soc., 1969, 91, 5675. 


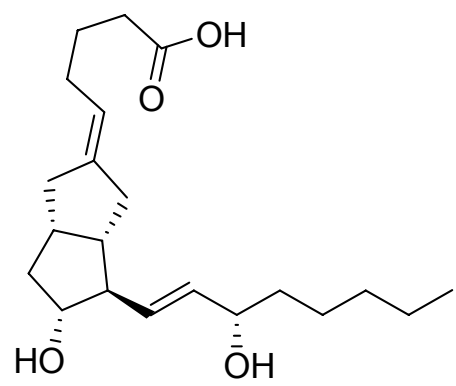

Carbacyclin (6)

$\mathrm{C}_{21} \mathbf{H}_{34} \mathbf{O}_{4} \quad \mathbf{M}_{\mathrm{r}}=350.25$

${ }^{1} \mathbf{H}$ NMR $\left(600 \mathrm{MHz}, \mathrm{CDCl}_{3}\right)$ 4.90-6.49 (1H, bs, COOH), 5.52 (1H, dd, $J$ 15.3, 7.0, vinylic in chain), 5.47 (1H, dd, $J 15.38 .3$, vinylic in chain), $5.22(1 \mathrm{H}, \mathrm{t}, J 6.7, \mathrm{C}=\mathrm{CH}), 4.05(1 \mathrm{H}, \mathrm{ddd}, J$ 6.9, 6.9, 6.6, $\mathrm{HC}=\mathrm{CHC}(\mathrm{OH}) H), 3.69(1 \mathrm{H}, \mathrm{ddd}, J$ 9.6, 9.6, 6.9, $\mathrm{HCOH}), 2.50-2.40(1 \mathrm{H}, \mathrm{m}$, $\left.\mathrm{CHCH}_{2} \mathrm{CHOH}\right), 2, .40-2.25$ (4H, m, alkyl $\left.\mathrm{CH}_{2}, \mathrm{CHHCHCH}, \mathrm{CH} \mathrm{CHCH}_{2} \mathrm{CHOH}\right), 2.18-1.99$ (5H, m, alkyl $\left.\mathrm{CH}_{2}, \mathrm{CH} H \mathrm{CHCH}, \mathrm{CHCHCHOH}\right), 1.97$ (1H, bd, $J$ 14.5, $\left.\mathrm{CHHCHCH}_{2} \mathrm{CHOH}\right)$, 1.82-1.73 (2H, m, alkyl $\left.\mathrm{CH}_{2}\right), 1.72-1.64(1 \mathrm{H}, \mathrm{m}, \mathrm{CHCHOH}), 1.62-1.53(1 \mathrm{H}, \mathrm{m}$, $\mathrm{C}(\mathrm{OH}) \mathrm{HCHH}), 1.51-1.43(1 \mathrm{H}, \mathrm{m}, \mathrm{C}(\mathrm{OH}) \mathrm{HCH} H), 1.40-1.22\left(6 \mathrm{H}, \mathrm{m}, 3\right.$ x alkyl $\left.\mathrm{CH}_{2}\right), 1.16$ (1H, ddd, $J 12.3,9.6,8.0, \mathrm{CHHCHCH}), 0.89\left(3 \mathrm{H}, \mathrm{t}, J 6.8, \mathrm{CH}_{3}\right)$

${ }^{13}$ C-NMR (100 MHz, $\left.\mathrm{CDCl}_{3}\right) 177.8$ (q), 142.7 (q), 135.3 (t), 133.5 (t), 120.9 (t), 77.2 (t), 73.4 $(\mathrm{t}), 56.9(\mathrm{t}), 45.1(\mathrm{t}), 41.5(\mathrm{~s}), 38.1(\mathrm{~s}), 37.4(\mathrm{t}), 36.9(\mathrm{~s}), 35.8(\mathrm{~s}), 32.5(\mathrm{~s}), 31.7(\mathrm{~s}), 28.4(\mathrm{~s})$, $25.2(\mathrm{~s}), 24.5(\mathrm{~s}), 22.6(\mathrm{~s}), 14.0(\mathrm{p})$

IR (Si, Film) $\widetilde{v}_{\max }=3306,2928,2858,1717,1684,1653,1558,1540,1507,1457,1033,971$

HRMS $\left(170{ }^{\circ} \mathrm{C} 70 \mathrm{eV}\right): \mathrm{m} / z$ calcd for $\mathrm{C}_{21} \mathrm{H}_{34} \mathrm{O}_{4}$ : 350.2457 ; found 350.2439

Optical Rotation: $[\alpha]_{\mathrm{D}}^{20}=+91.4\left(\mathrm{c}=0.64, \mathrm{CH}_{3} \mathrm{OH}\right)$

$\boldsymbol{R}_{\mathbf{f}}(\mathrm{EtOAc} / 1 \% \mathrm{AcOH})=0.33$

${ }^{1} \mathrm{H},{ }^{13} \mathrm{C}$ NMR and optical rotation were concurrent with literature values for Carbacyclin. See a) D. R. Morton, Jr.; F. C. Brokaw, J. Org. Chem. 1979, 44, 2880; b) S. Okamoto; K. Subburaj; F. Sato, J. Am. Chem. Soc. 2000, 122, 11244. 


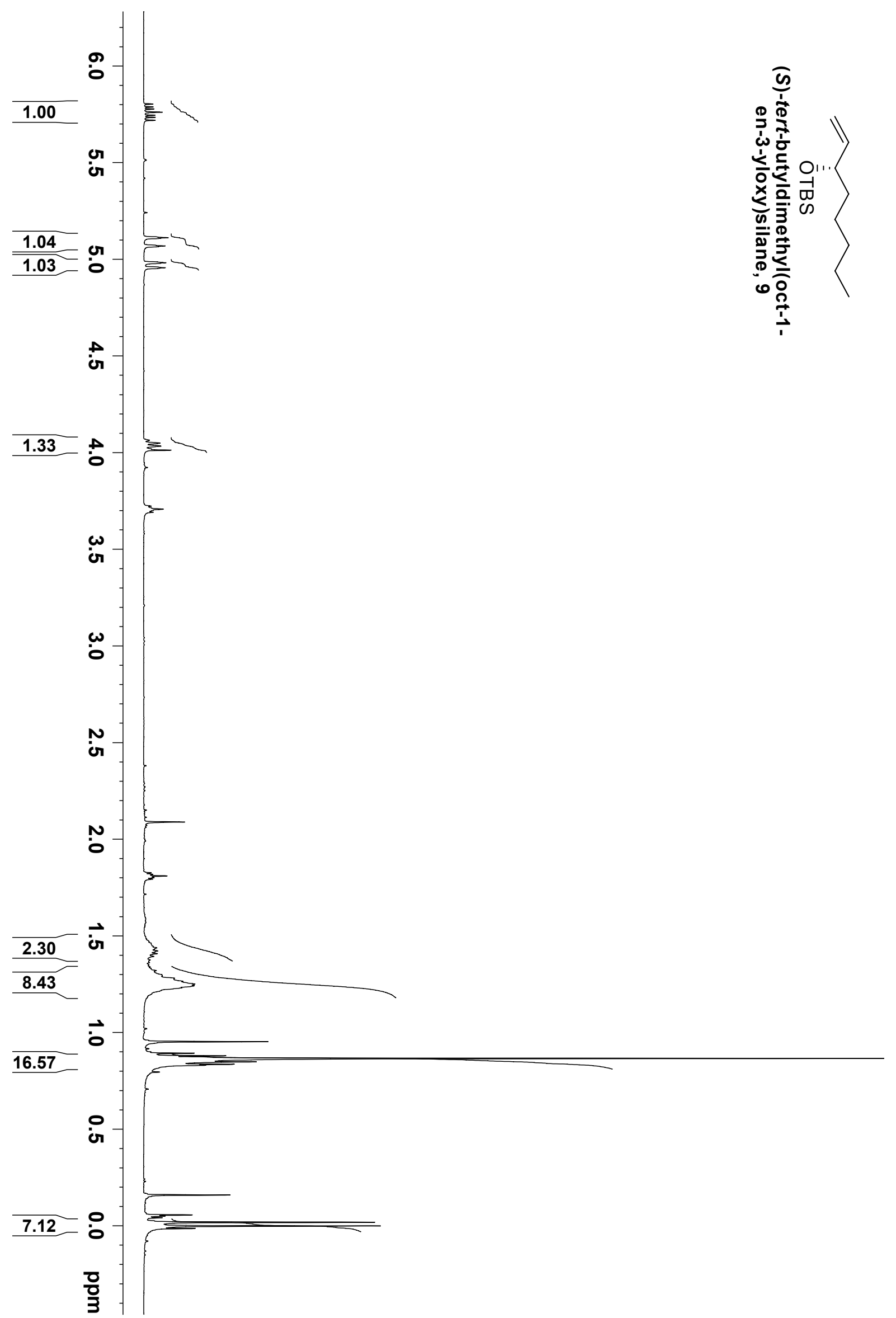




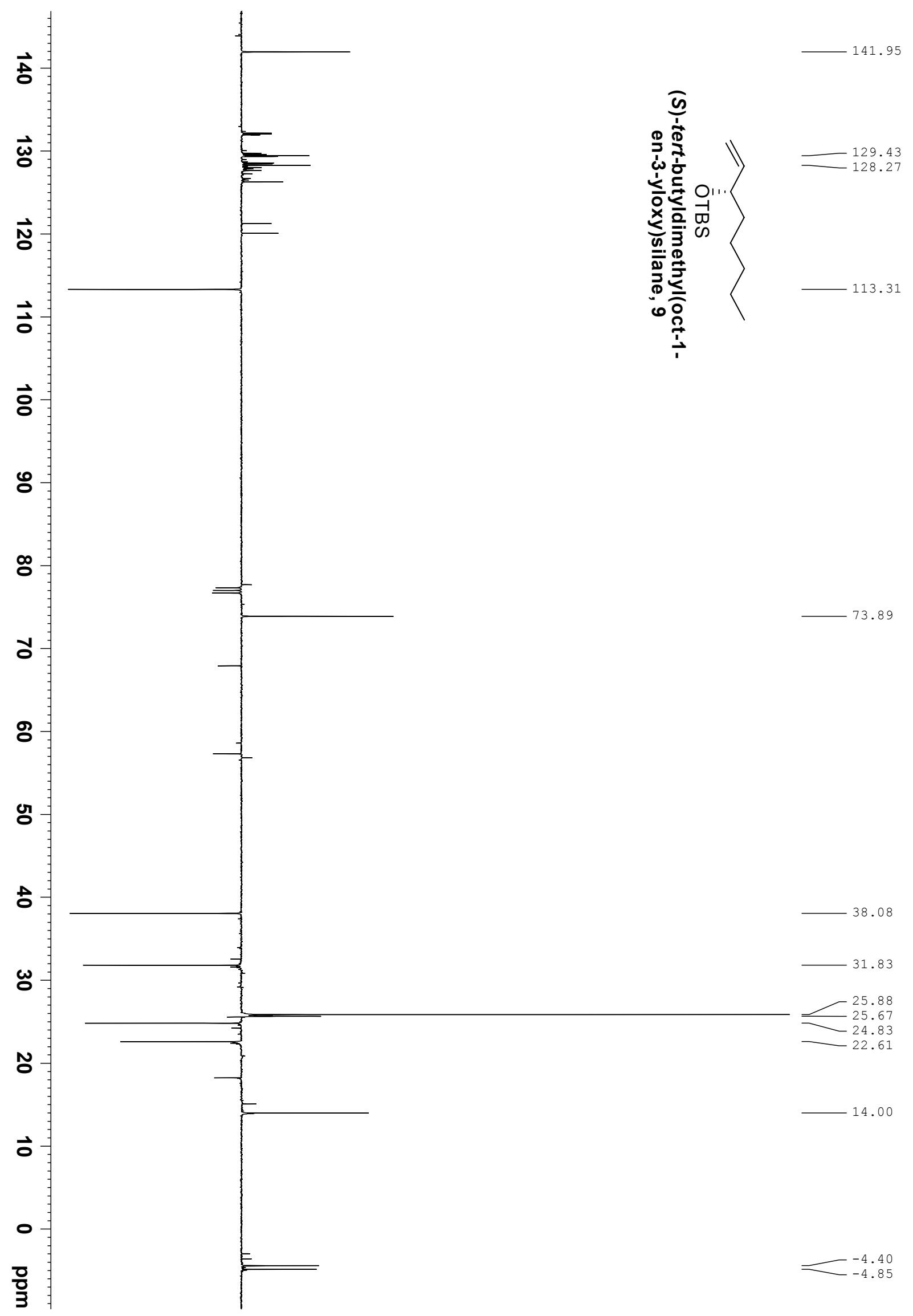




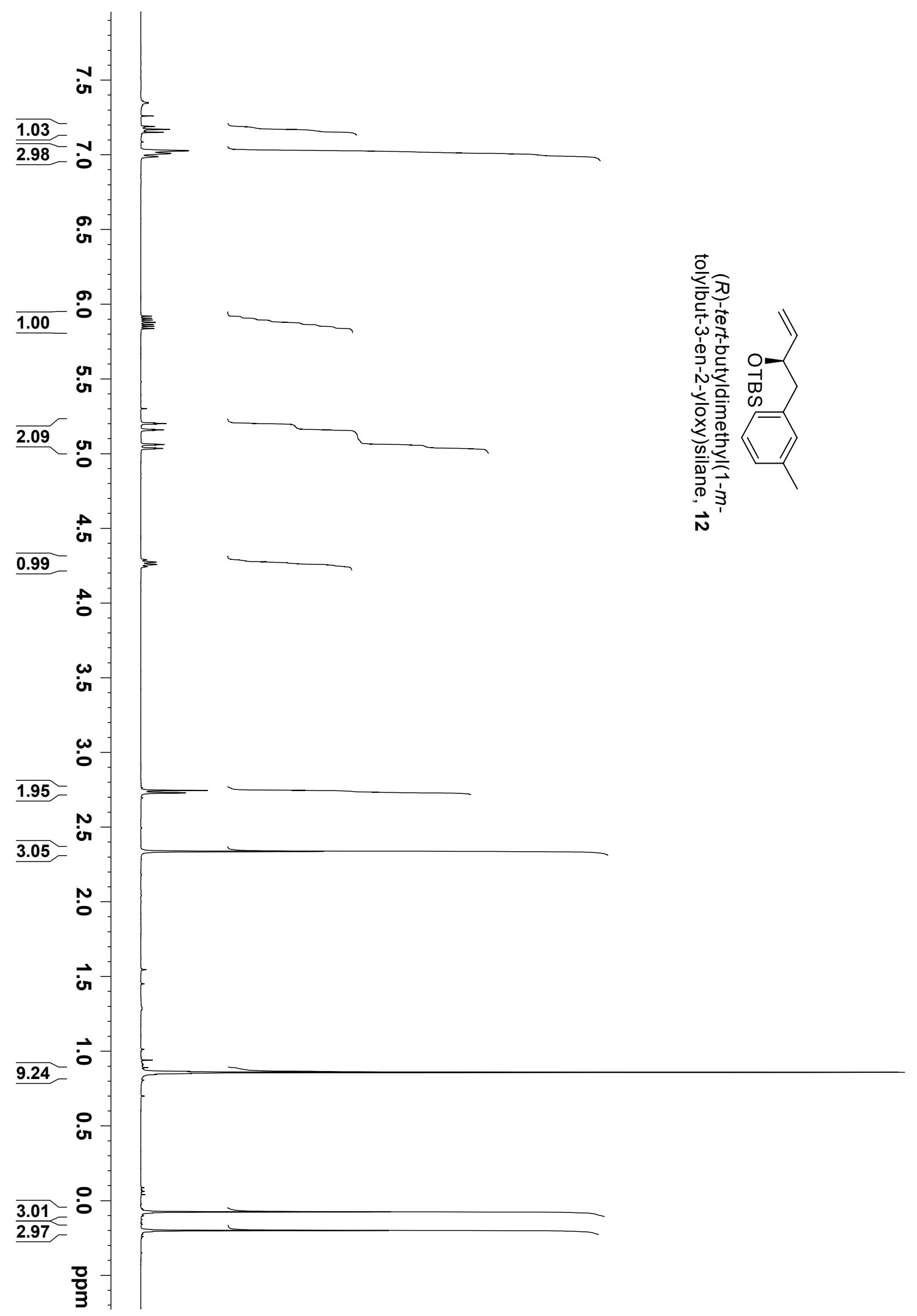




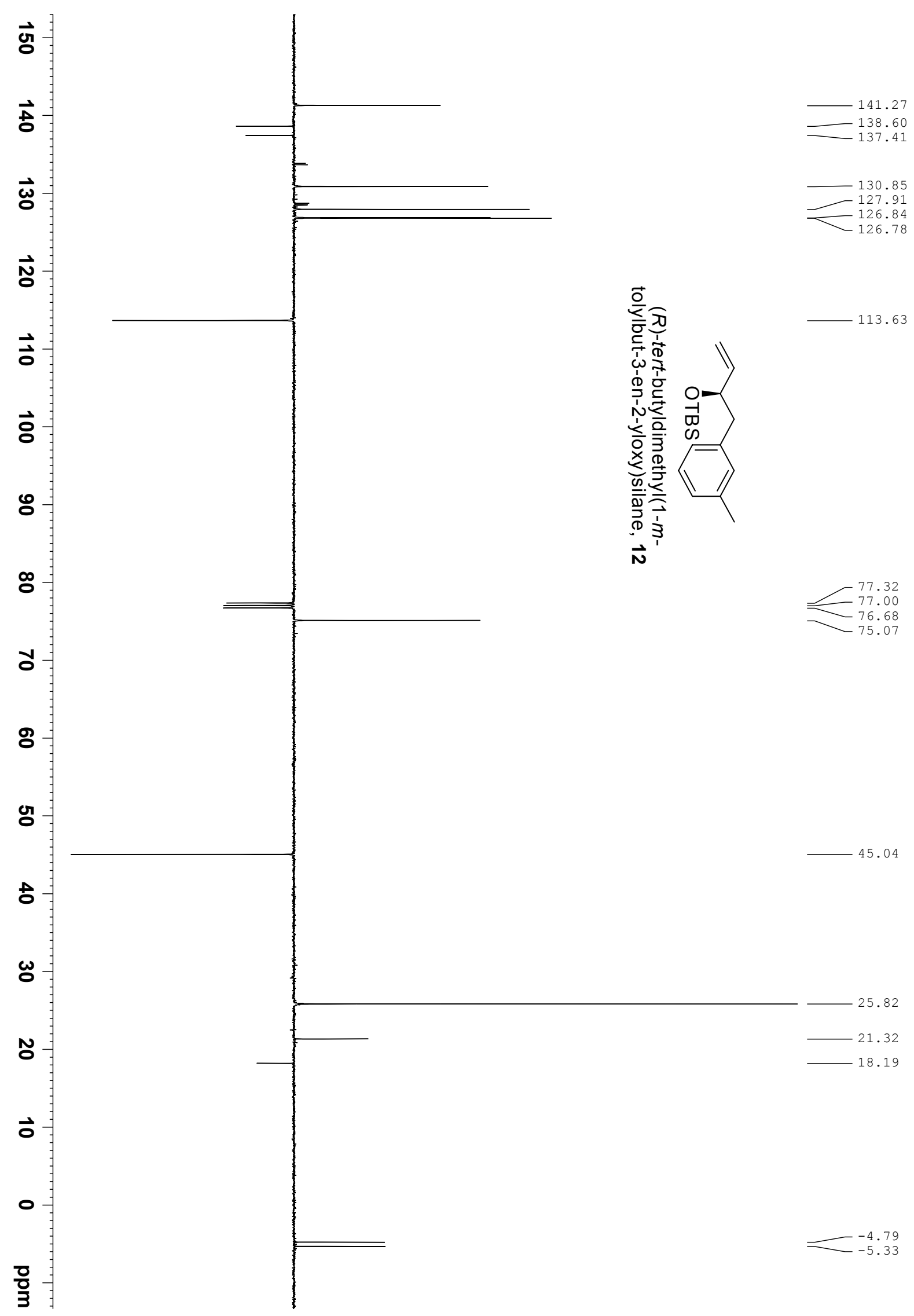




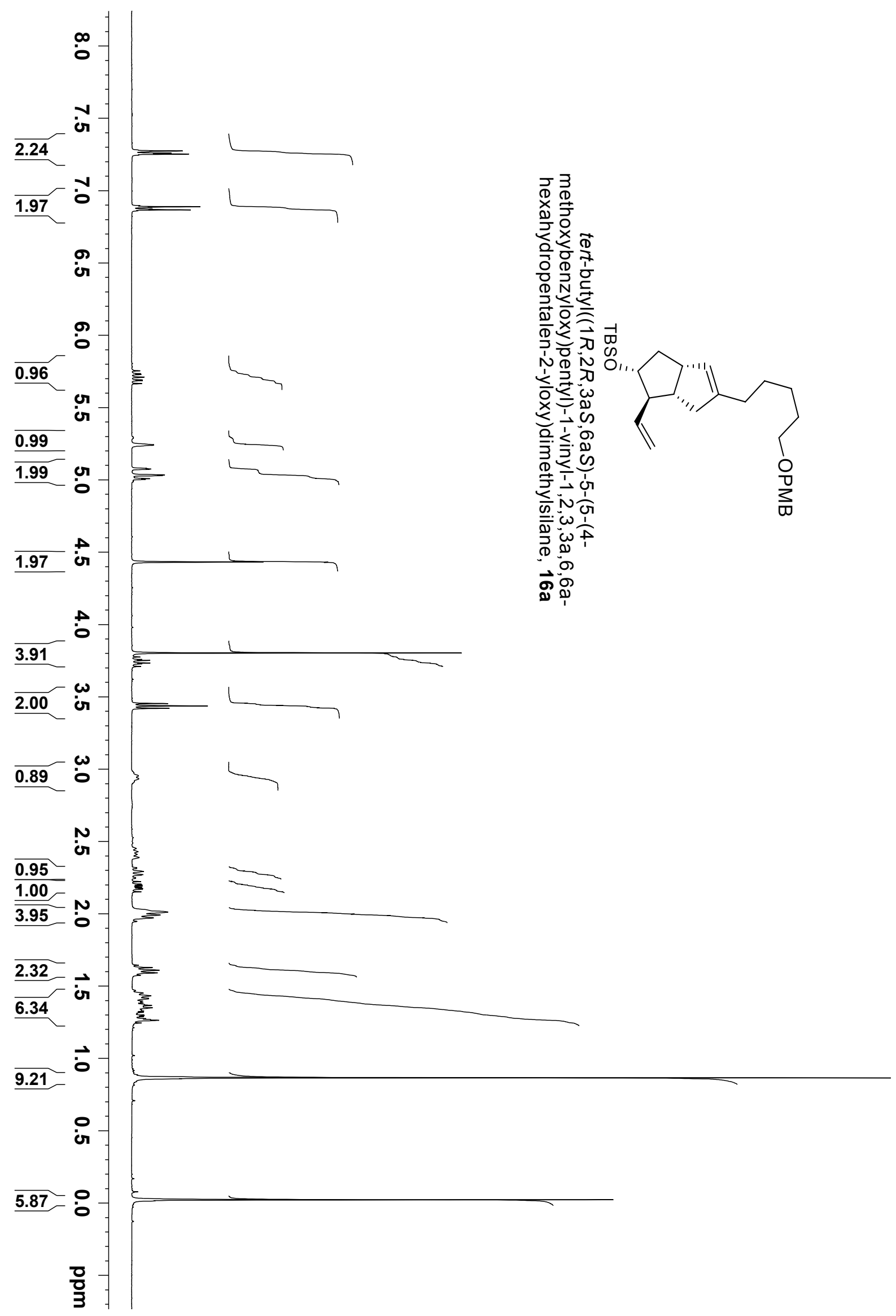




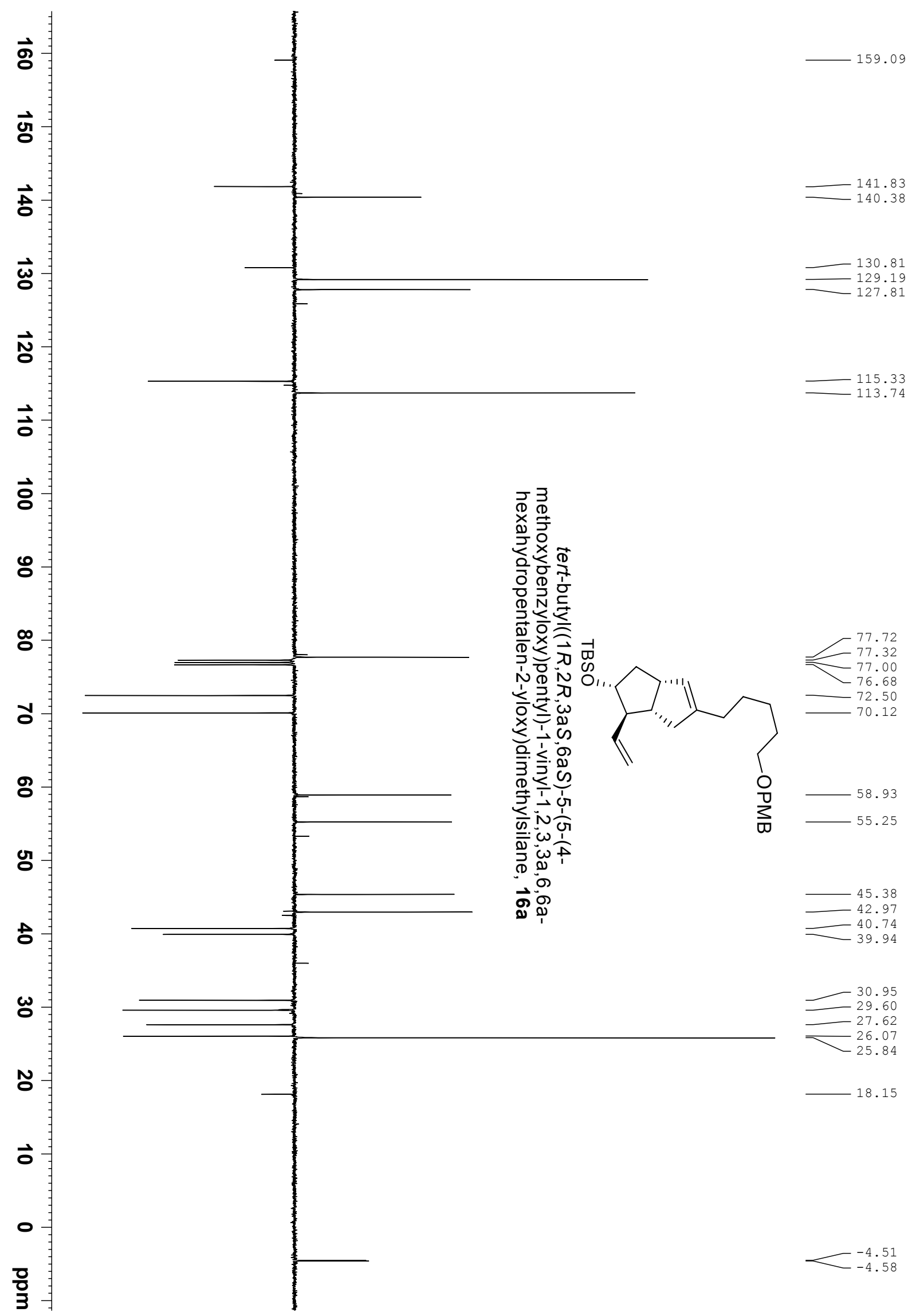




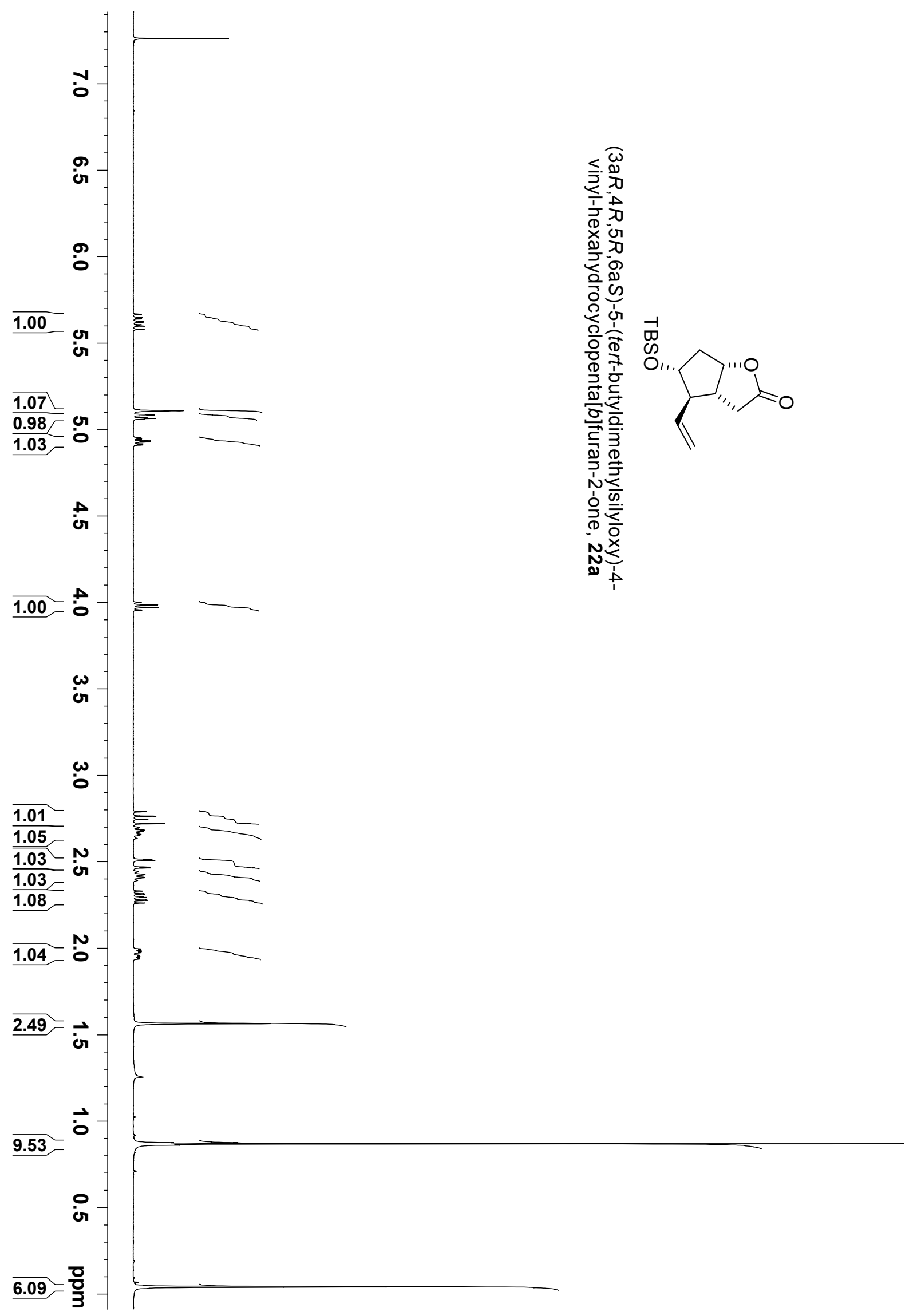




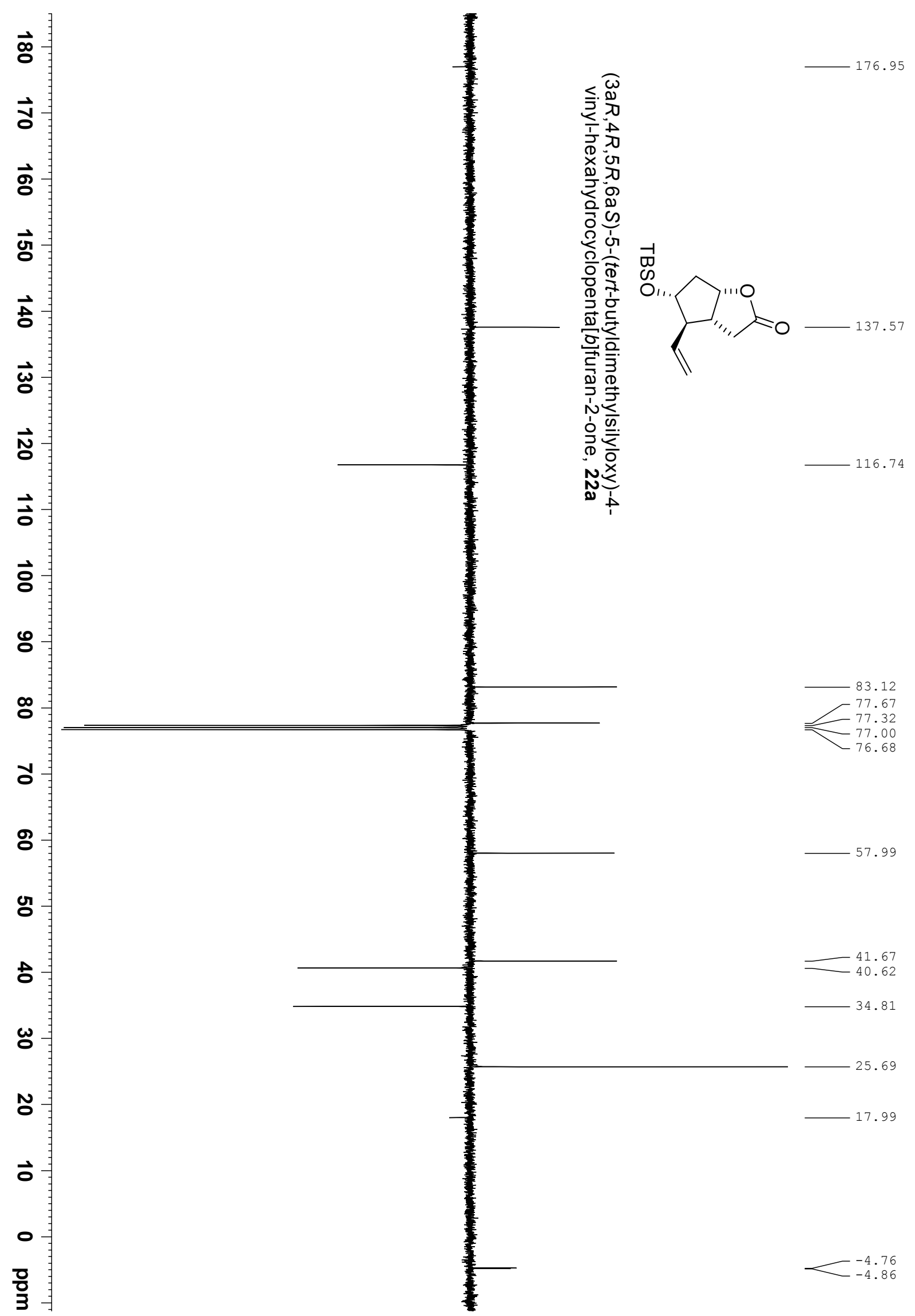




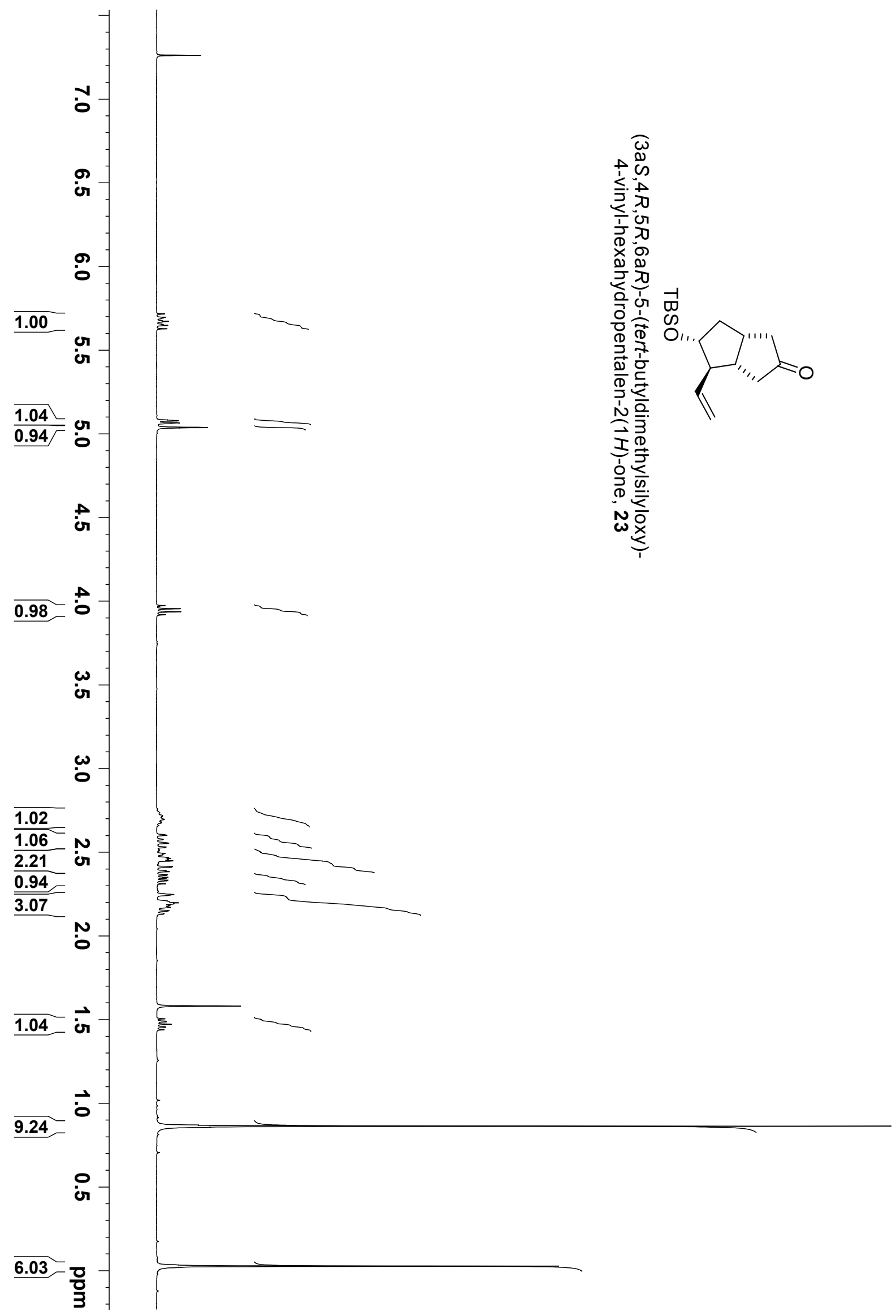




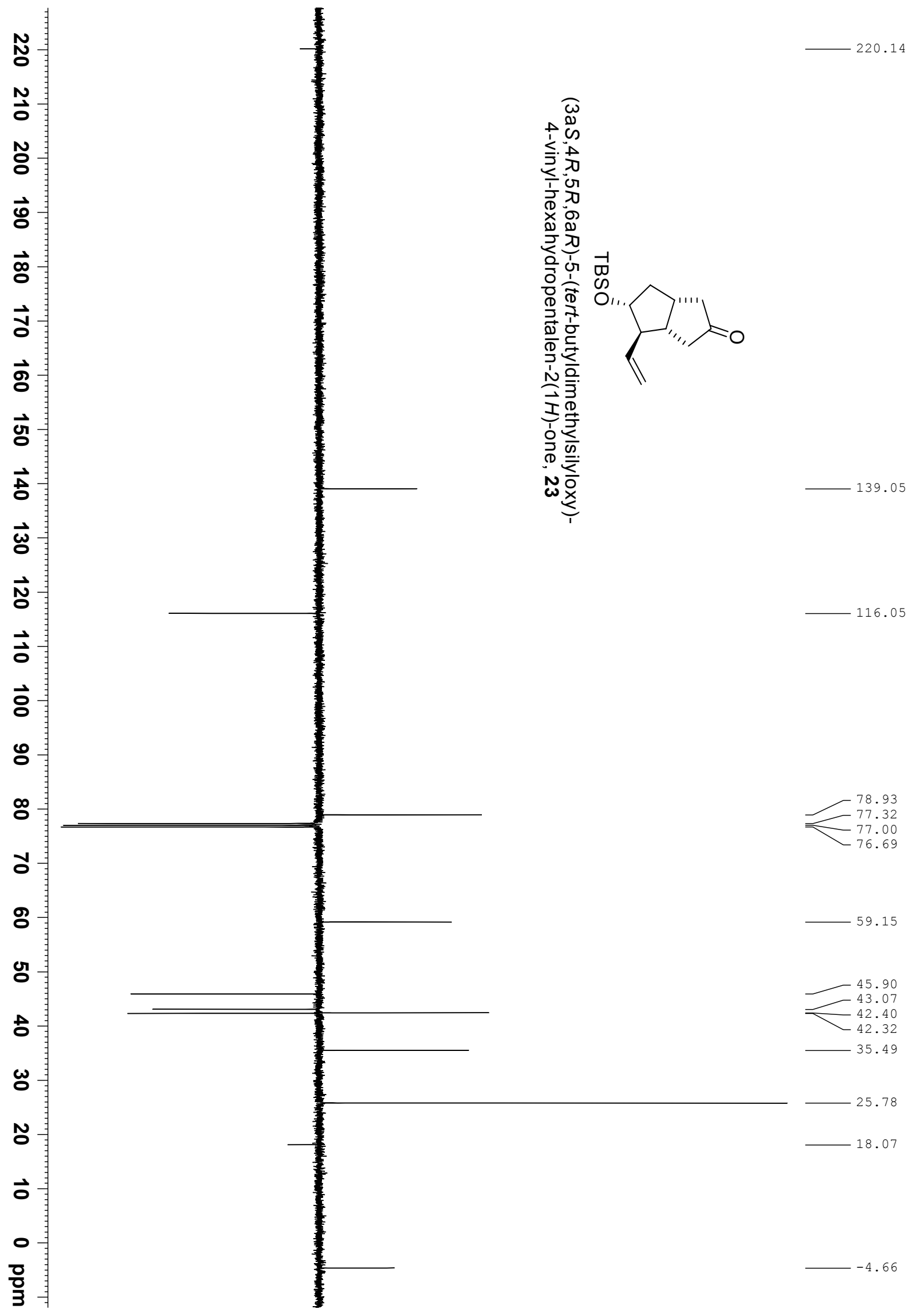

30 


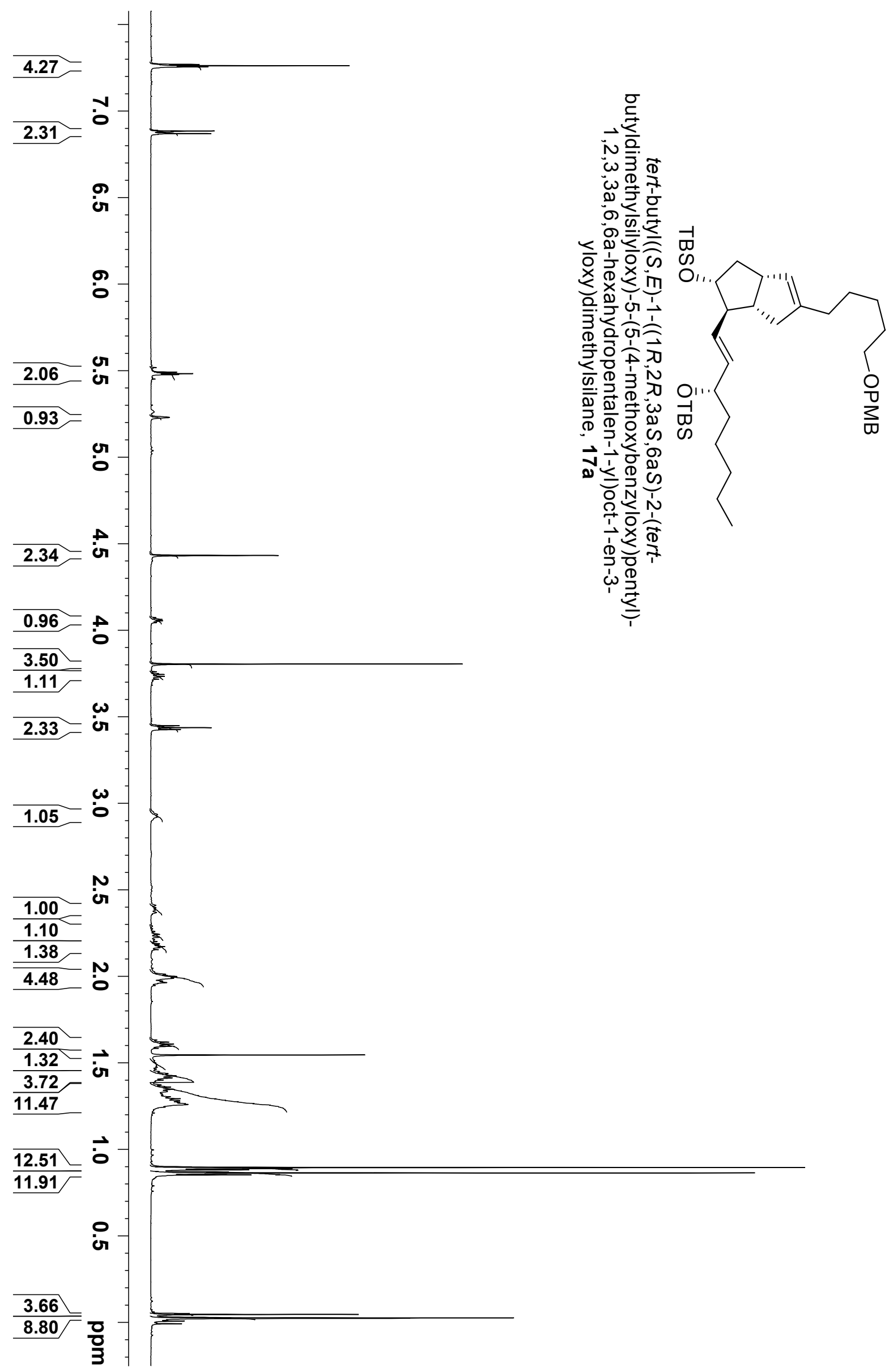




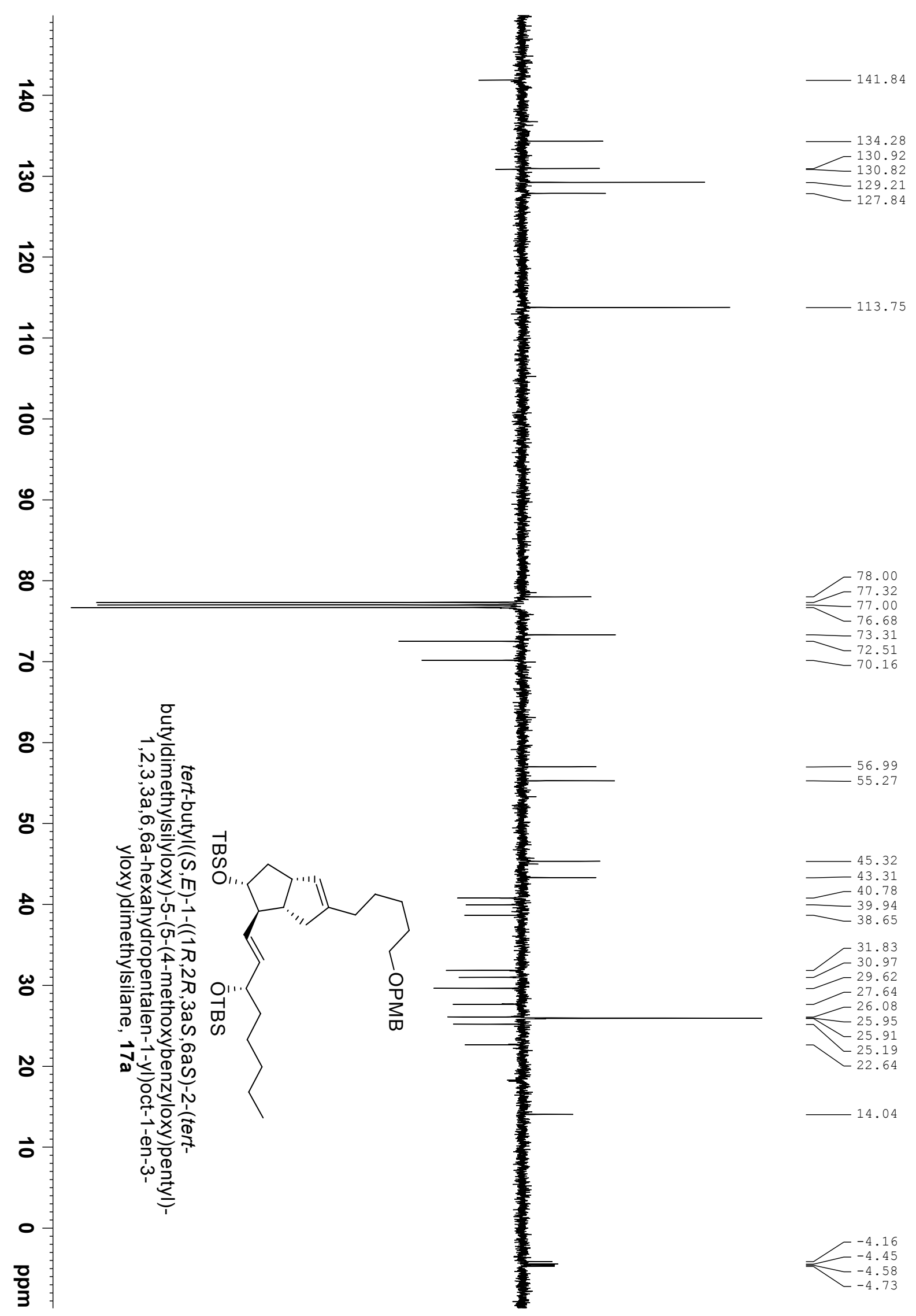




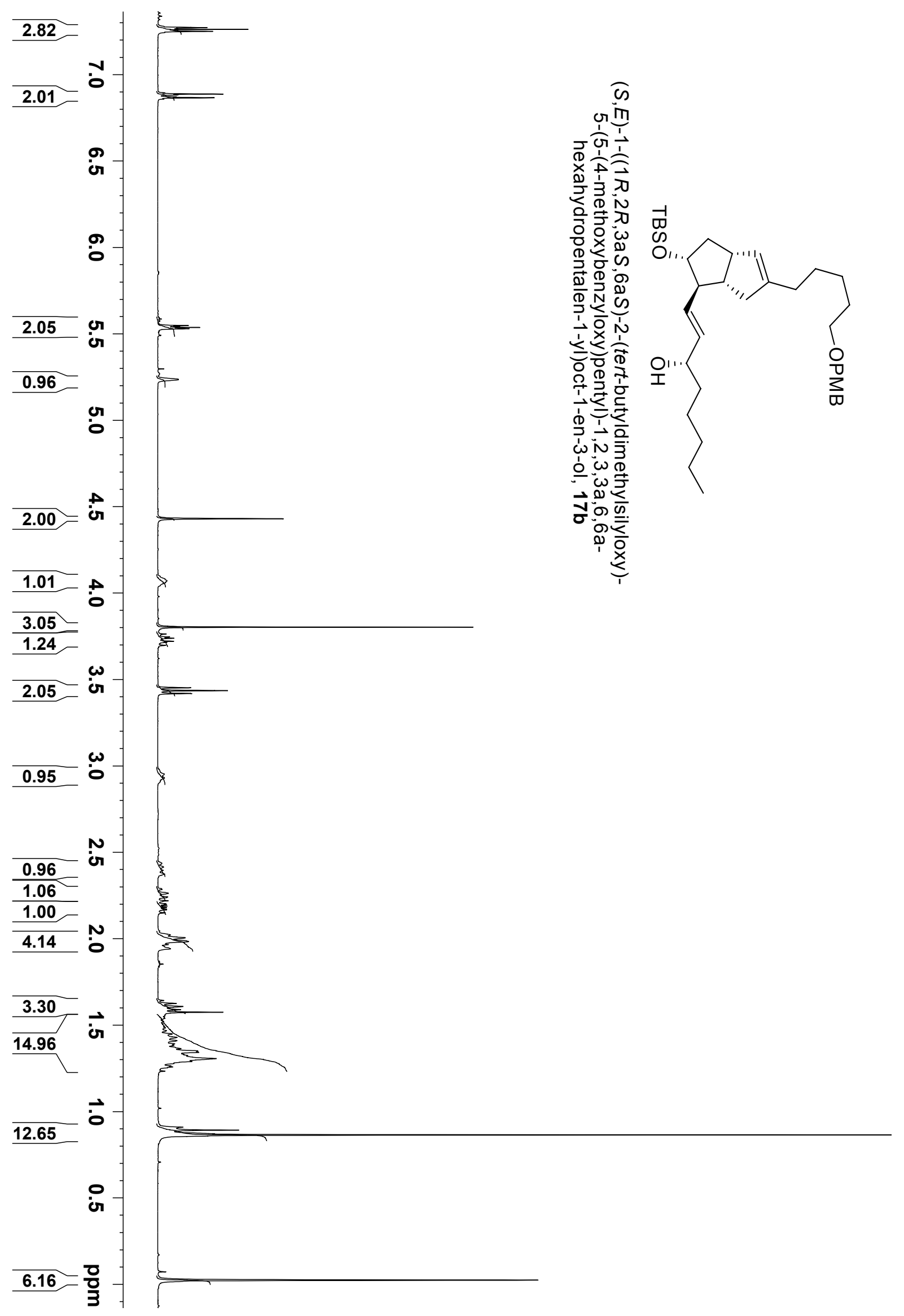



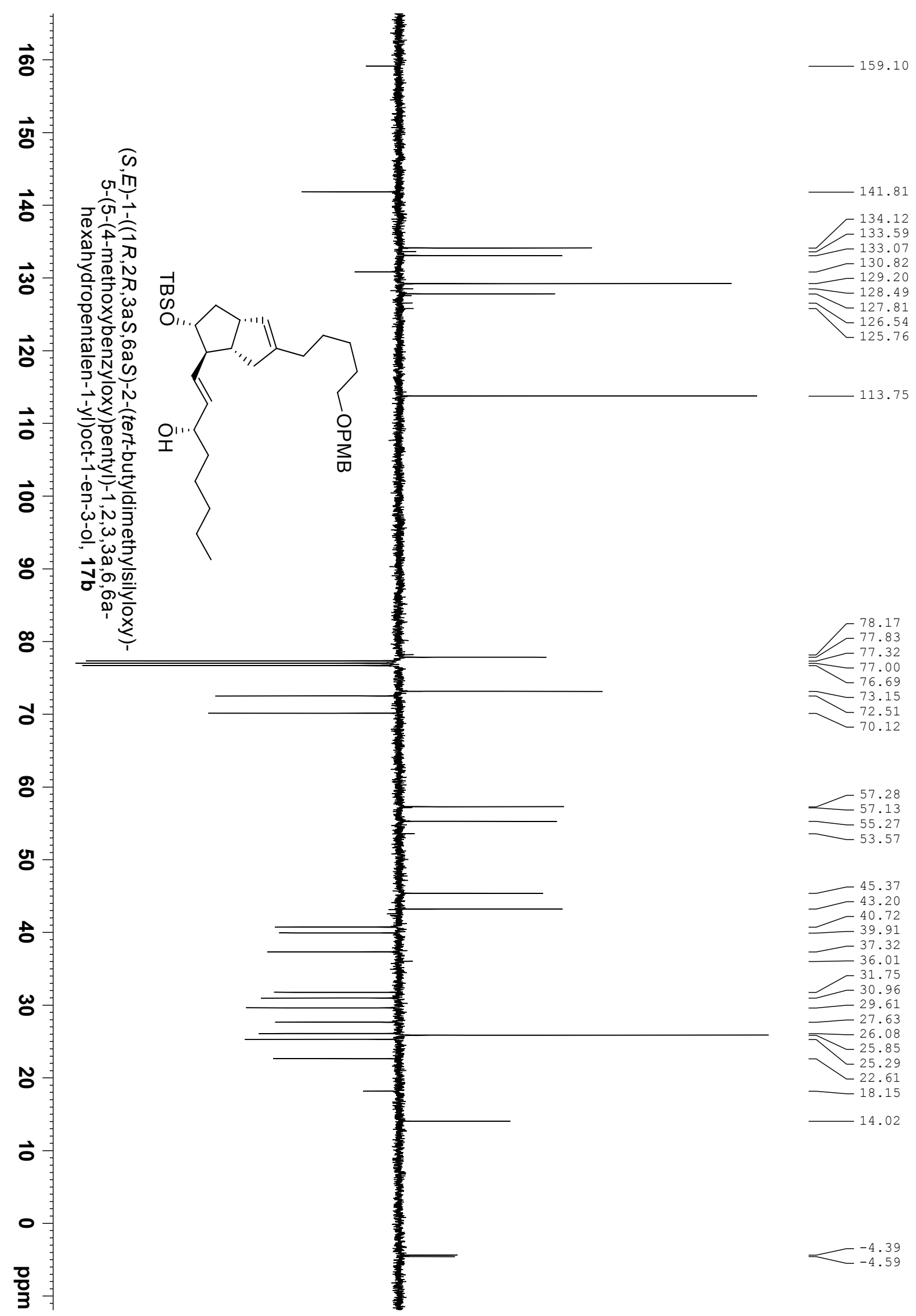


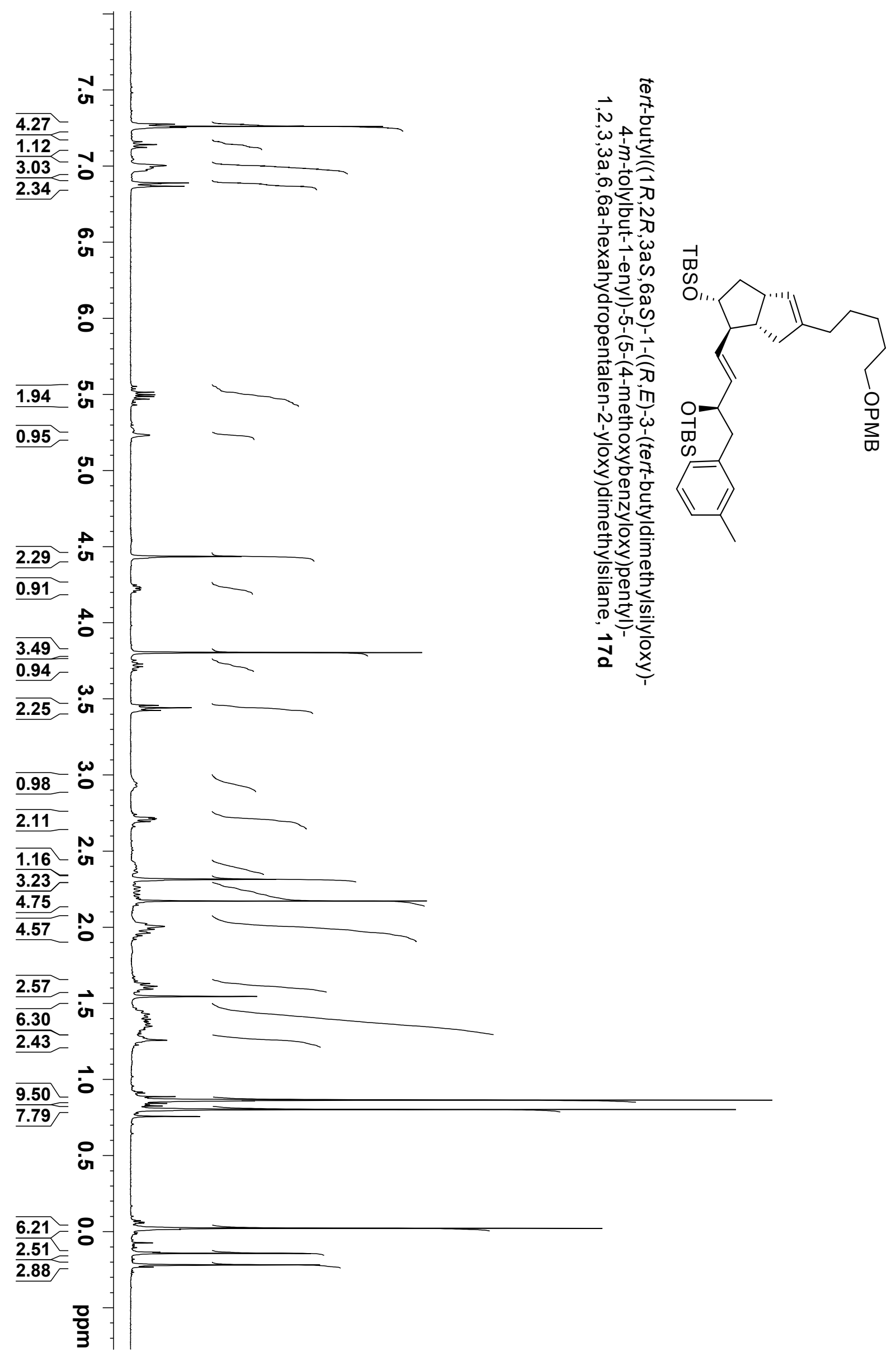



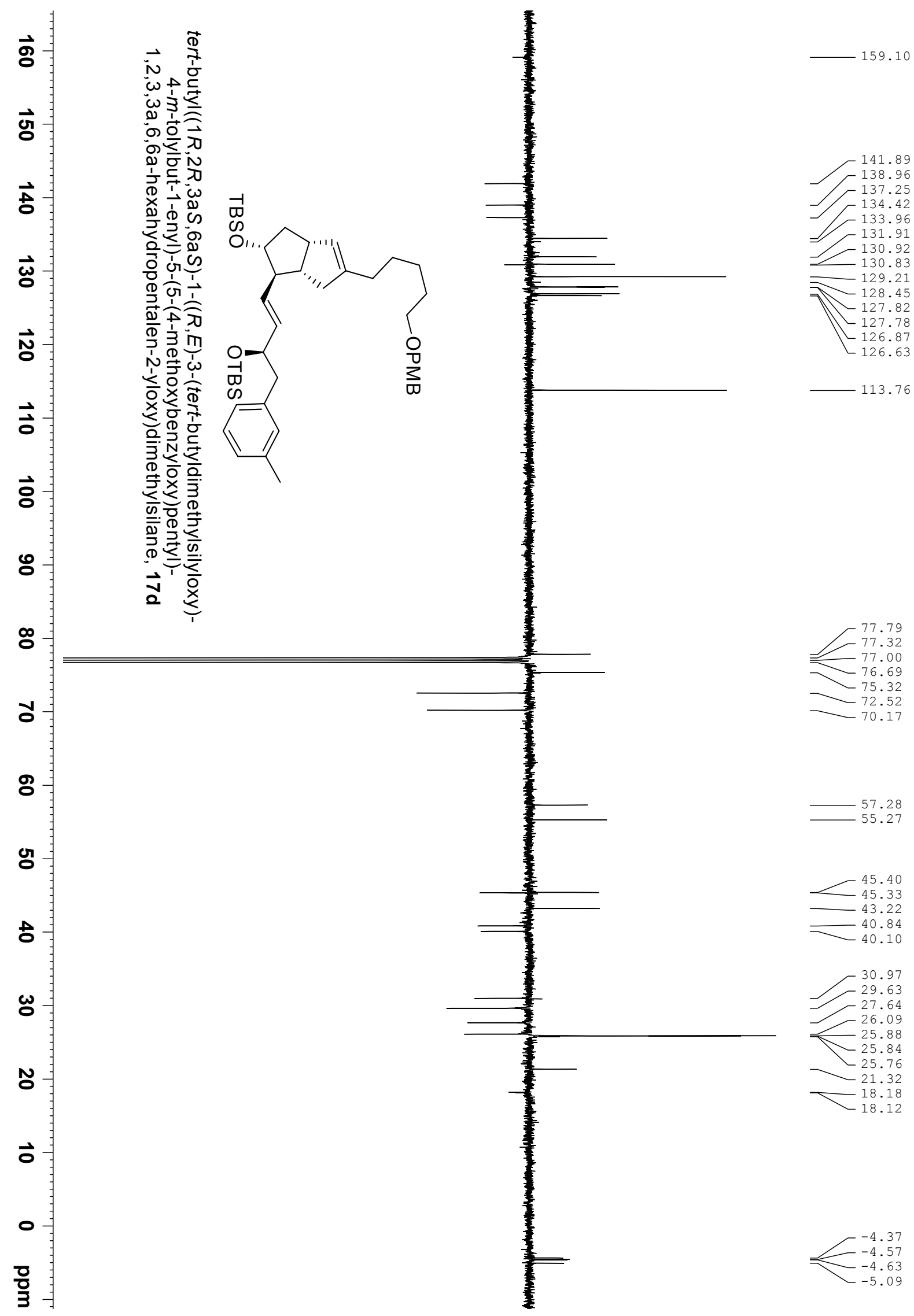

57.28 55.27
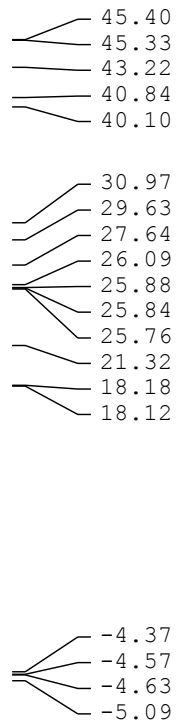


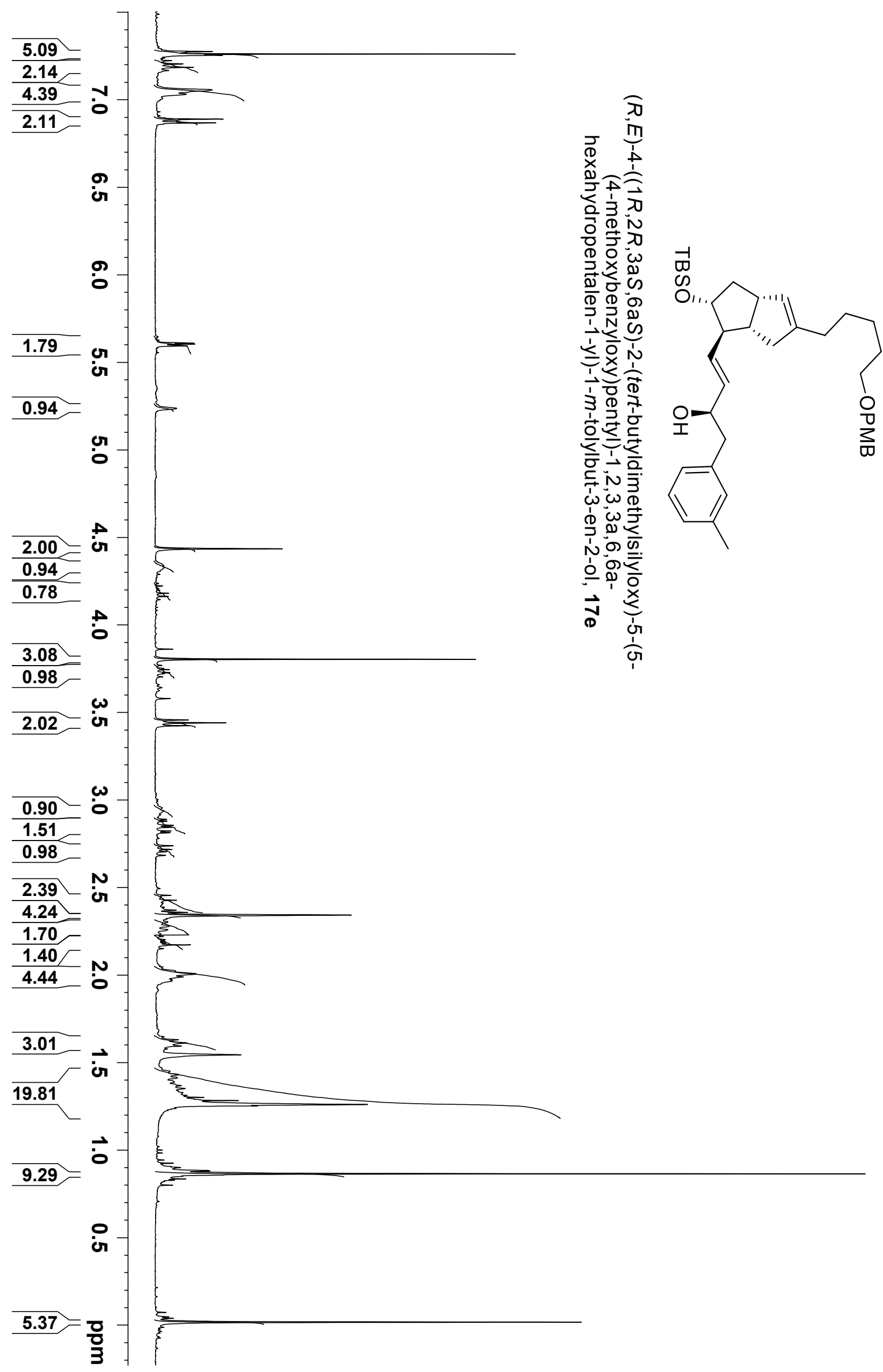




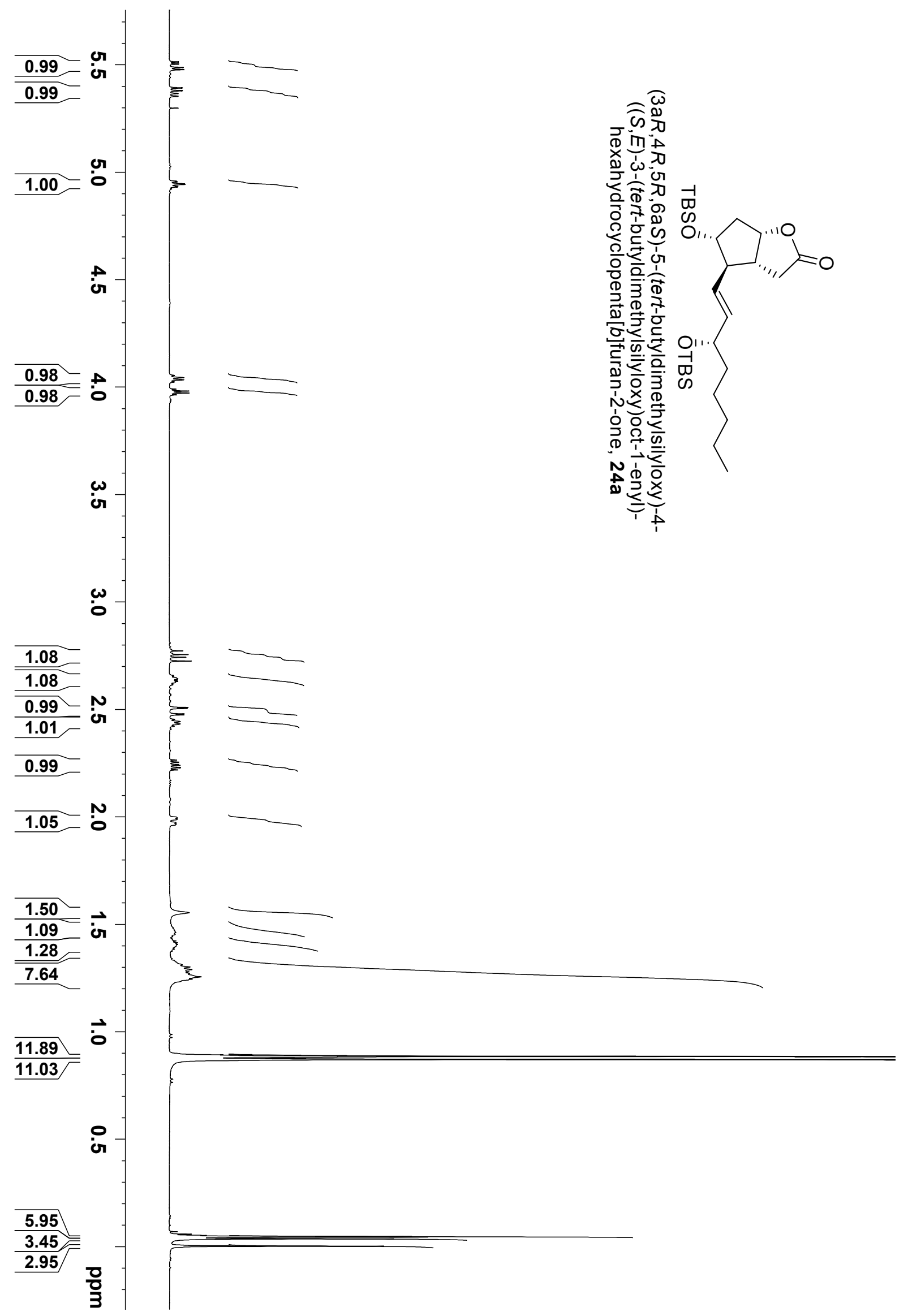



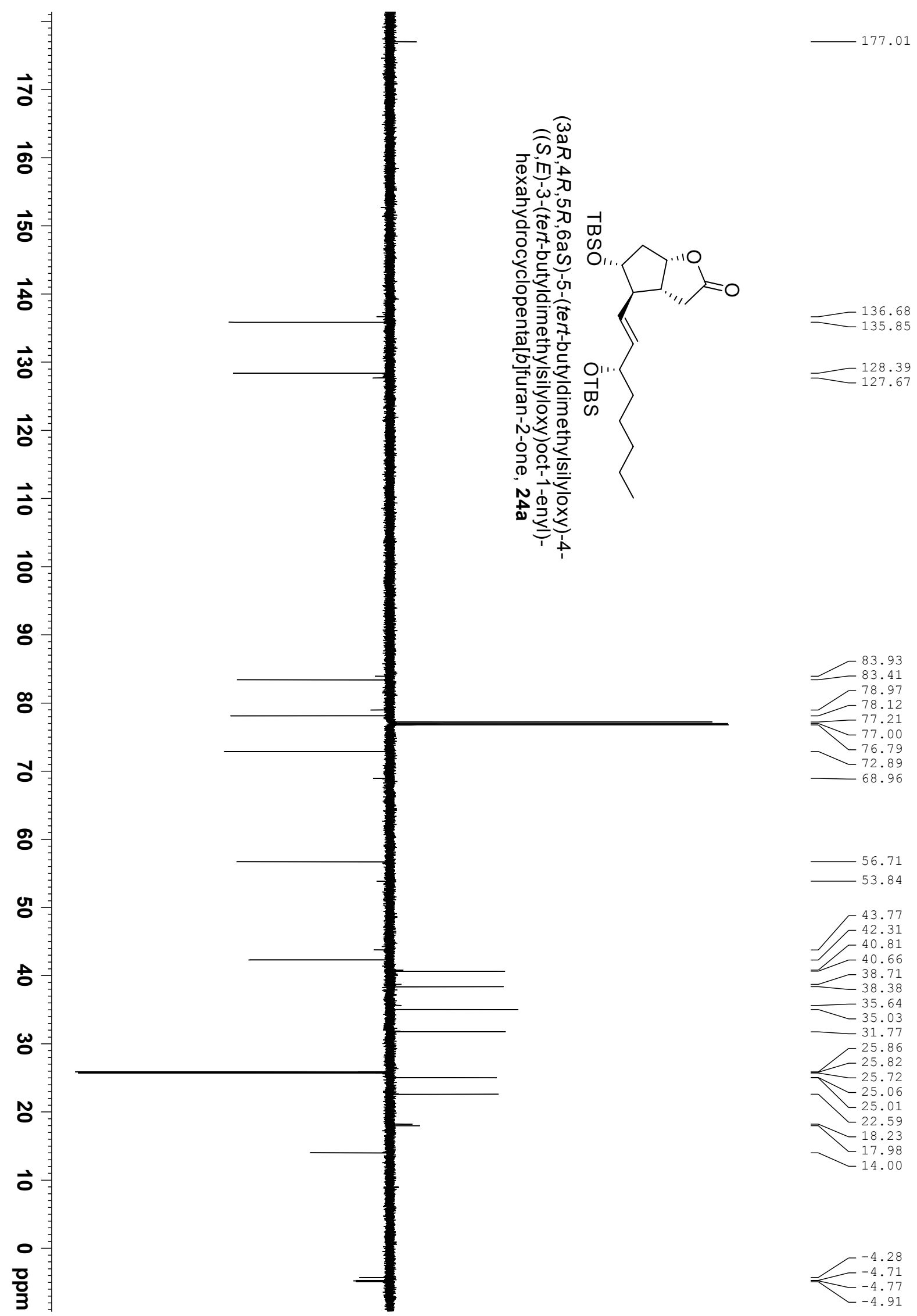


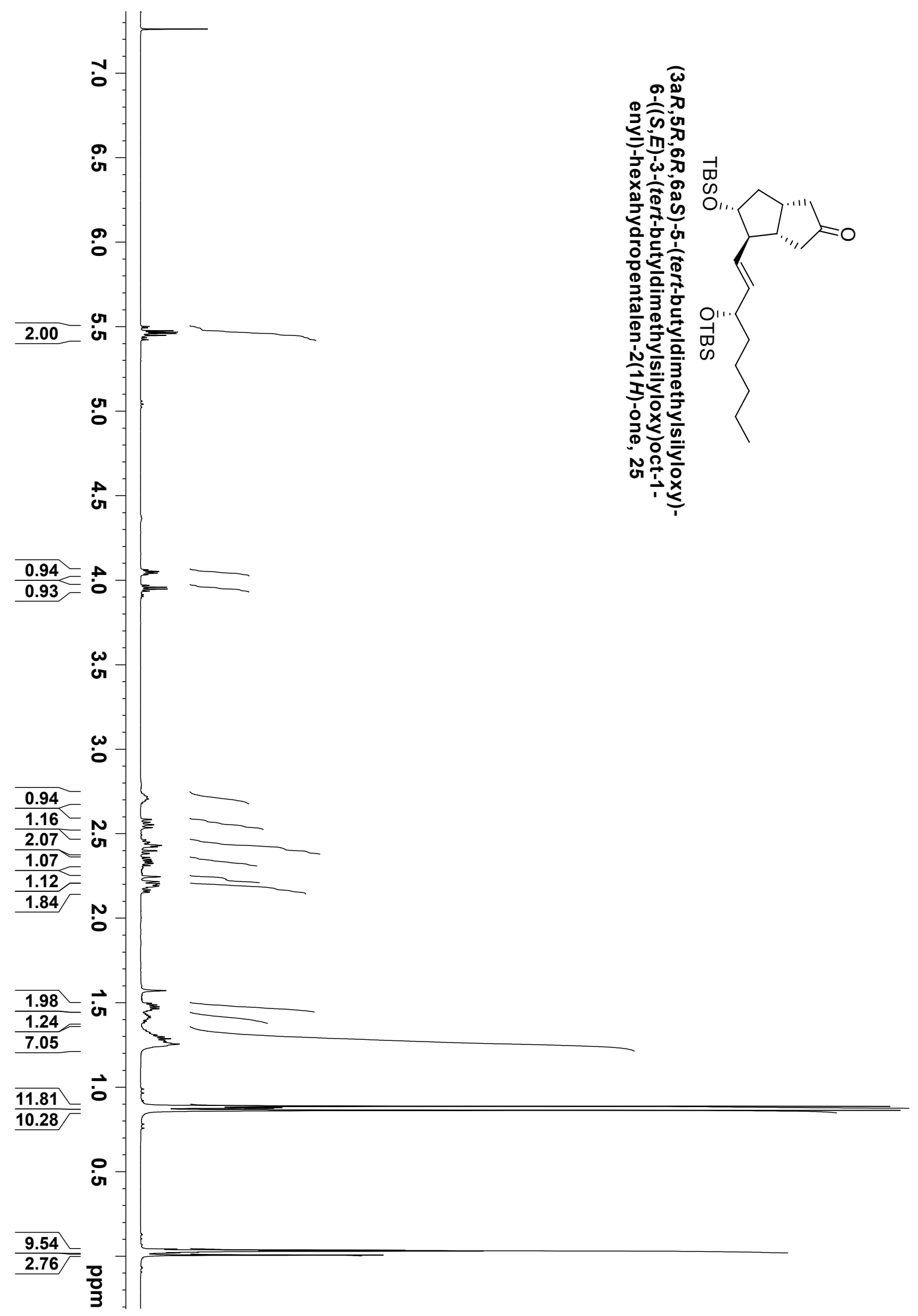




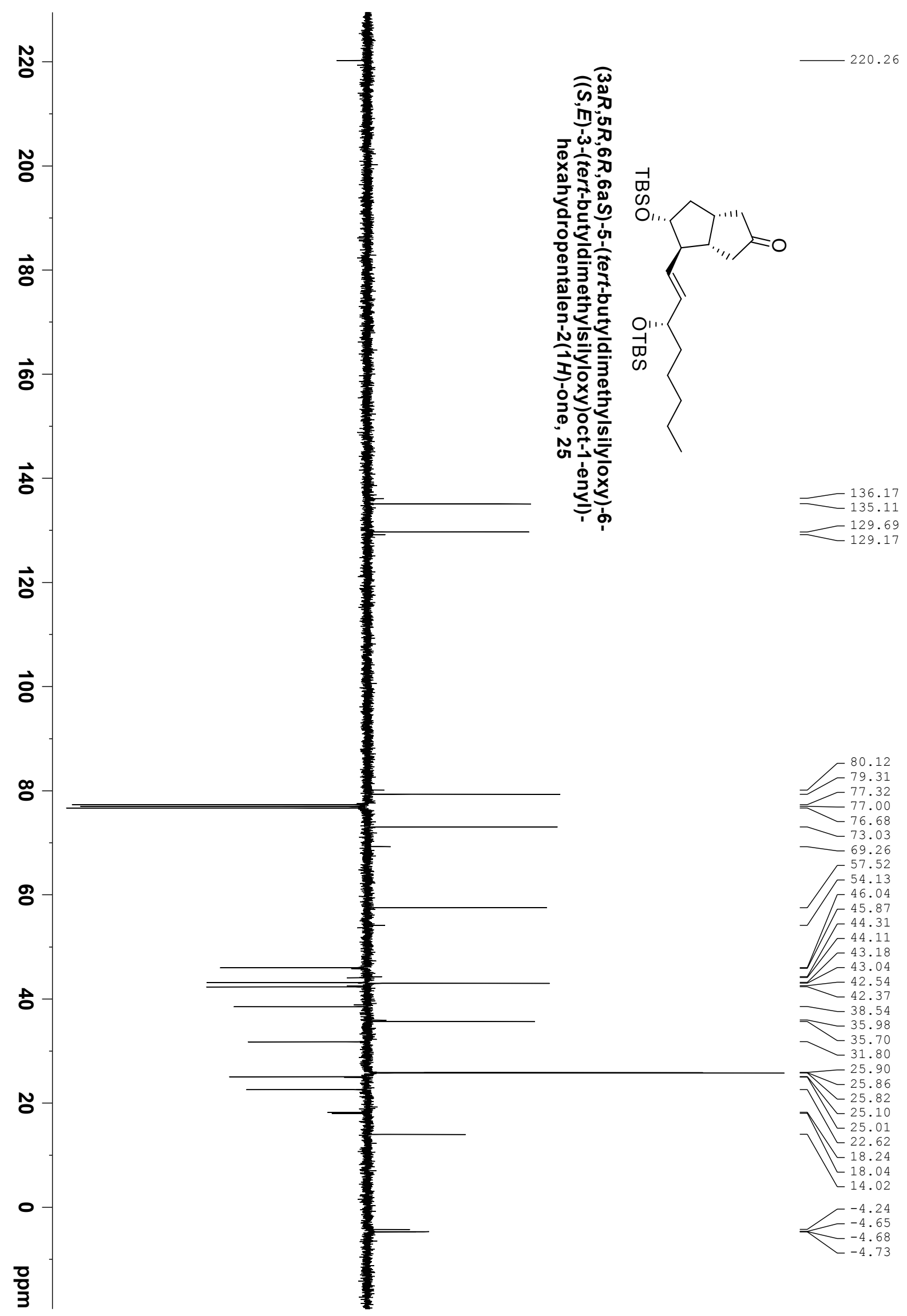



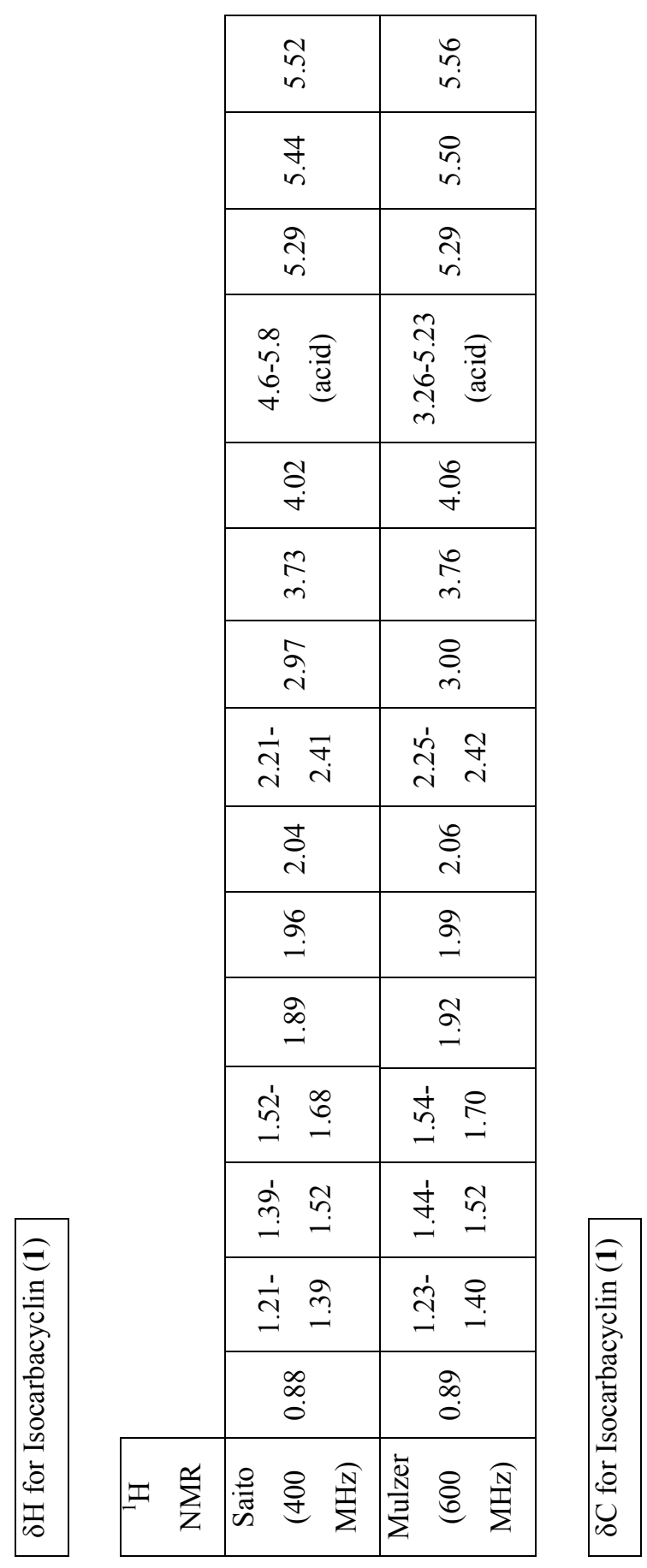

\begin{tabular}{|c|c|c|}
\hline & 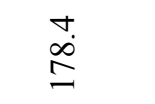 & $\vec{\infty}$ \\
\hline & $\stackrel{\circ}{\stackrel{\dot{\Xi}}{ }}$ & 守 \\
\hline & $\begin{array}{l}0 \\
\ddot{n} \\
\ddot{n}\end{array}$ & $\begin{array}{l}\dot{0} \\
\dot{m} \\
\ddot{n}\end{array}$ \\
\hline & $\stackrel{n}{m}$ & 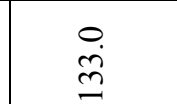 \\
\hline & $\begin{array}{l}\infty \\
\infty \\
\stackrel{\infty}{\bigcup}\end{array}$ & $\begin{array}{l}\stackrel{\leftrightarrow}{\infty} \\
\stackrel{\infty}{\simeq}\end{array}$ \\
\hline & $\vec{r}$ & 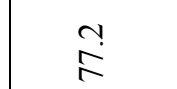 \\
\hline & $\ddot{n}$ & $\ddot{n}$ \\
\hline & $\frac{a}{i n}$ & $\begin{array}{l}0 \\
\infty \\
i\end{array}$ \\
\hline & $\underset{⿱ 亠 凶}{\dot{q}}$ & $\begin{array}{l}\dot{0} \\
\ddot{f}\end{array}$ \\
\hline & $\stackrel{\stackrel{Y}{f}}{\underset{f}{*}}$ & $\stackrel{m}{f}$ \\
\hline & ले & $\stackrel{n}{\text { mे }}$ \\
\hline & $\overrightarrow{\text { mे }}$ & $\stackrel{\sim}{\grave{n}}$ \\
\hline & 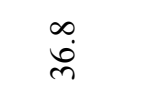 & $\underset{m}{\stackrel{0}{m}}$ \\
\hline & ले & $\stackrel{\vec{m}}{m}$ \\
\hline & $\stackrel{i}{m}$ & i. \\
\hline & ?ి & $\stackrel{?}{\text { ?. }}$ \\
\hline & $\begin{array}{l}0 \\
\stackrel{\sim}{0}\end{array}$ & ڤ్రి \\
\hline & ڤั) & $\stackrel{\text { ָे }}{\sim}$ \\
\hline & $\stackrel{?}{\sim}$ & $\begin{array}{l}0 \\
\stackrel{\sim}{\sim}\end{array}$ \\
\hline & $\begin{array}{l}0 \\
\text { ì }\end{array}$ & $\begin{array}{l}0 \\
i \\
i\end{array}$ \\
\hline & $\stackrel{\circ}{\stackrel{\circ}{ \pm}}$ & $\stackrel{\circ}{\stackrel{+}{ \pm}}$ \\
\hline & 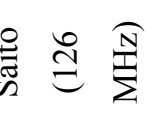 & 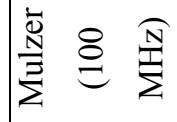 \\
\hline
\end{tabular}




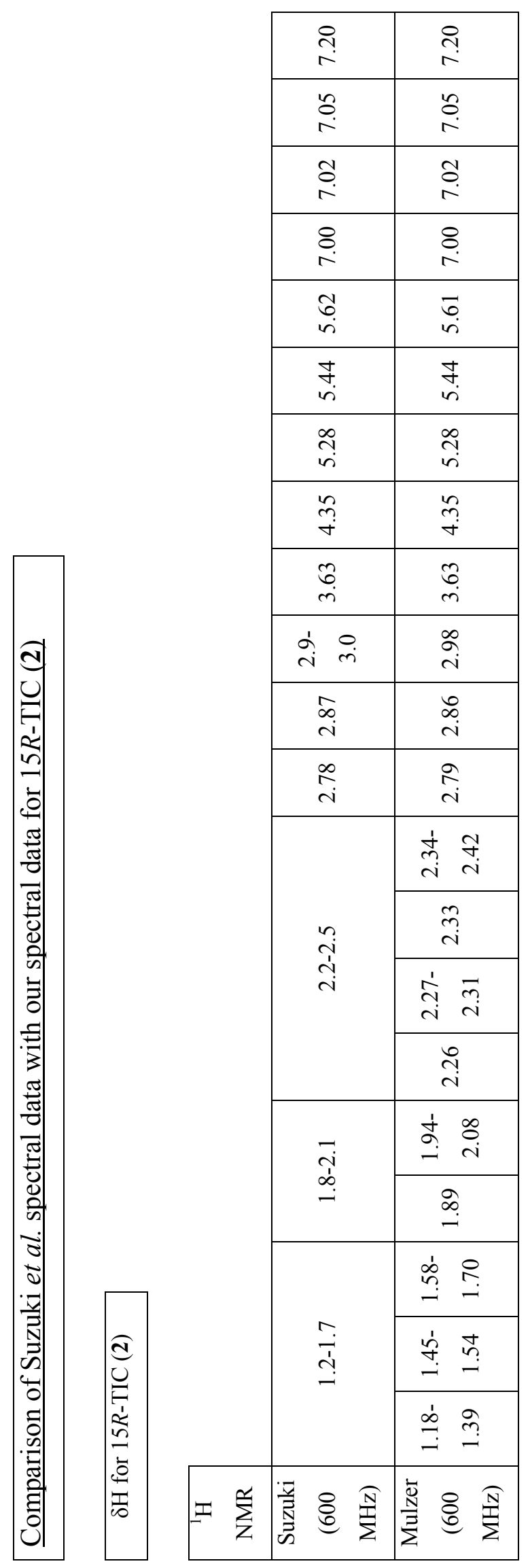

\begin{tabular}{|c|c|}
\hline$\underset{\infty}{\infty}$ & $\underset{\infty}{\infty}$ \\
\hline$\stackrel{\Xi}{\dot{\Xi}}$ & $\stackrel{n}{\dot{\Xi}}$ \\
\hline 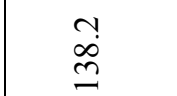 & $\underset{\substack{n \\
\infty \\
\infty}}{0}$ \\
\hline 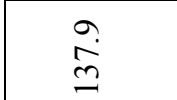 & $\stackrel{\infty}{\stackrel{\infty}{n}}$ \\
\hline$\stackrel{m}{\stackrel{m}{m}}$ & $\stackrel{m}{\stackrel{m}{m}}$ \\
\hline 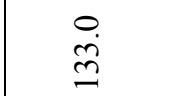 & 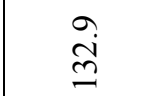 \\
\hline 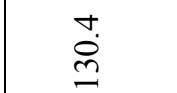 & 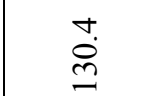 \\
\hline $\begin{array}{l}0 \\
\dot{0} \\
\stackrel{\nearrow}{\nearrow}\end{array}$ & 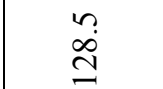 \\
\hline$\underset{\substack{+ٍ}}{\stackrel{\sim}{\sim}}$ & $\begin{array}{l}m \\
\stackrel{\infty}{0} \\
\stackrel{\sim}{\simeq}\end{array}$ \\
\hline 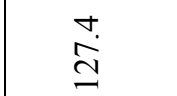 & $\stackrel{\Xi}{\stackrel{\Xi}{\Xi}}$ \\
\hline 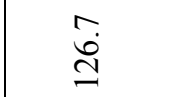 & $\begin{array}{l}\stackrel{0}{0} \\
\stackrel{\sim}{\beth}\end{array}$ \\
\hline$\stackrel{?}{\stackrel{2}{n}}$ & $\stackrel{?}{\stackrel{2}{r}}$ \\
\hline$\ddot{r}$ & 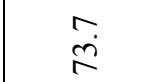 \\
\hline $\begin{array}{c}\text { No } \\
\infty \\
i n\end{array}$ & $\vec{\infty}$ \\
\hline$\ddot{i}$ & $\begin{array}{l}\dot{b} \\
\ddot{f}\end{array}$ \\
\hline$\stackrel{n}{\text { f }}$ & $\stackrel{n}{7}$ \\
\hline 导 & $\overline{\dot{f}}$ \\
\hline ڤ̊. & ڤn \\
\hline$\stackrel{\text { ڤे }}{m}$ & $\stackrel{m}{\text { mे }}$ \\
\hline लि & $\tilde{m}$ \\
\hline & 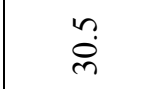 \\
\hline 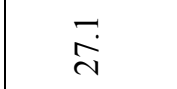 & $\stackrel{\circ}{\stackrel{\sim}{\sim}}$ \\
\hline$\stackrel{+}{\stackrel{+}{\sim}}$ & $\stackrel{+}{\stackrel{+}{\sim}}$ \\
\hline$\stackrel{n}{\grave{N}}$ & $\stackrel{+}{\dot{N}}$ \\
\hline 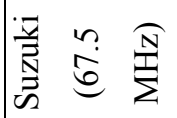 & 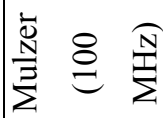 \\
\hline
\end{tabular}




\begin{tabular}{|c|c|c|}
\hline & & 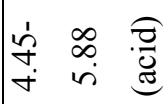 \\
\hline & $\begin{array}{l}n \\
n \\
n \\
n\end{array}$ & $\begin{array}{l}\infty \\
i n \\
i n\end{array}$ \\
\hline \multirow{3}{*}{\multicolumn{2}{|c|}{ 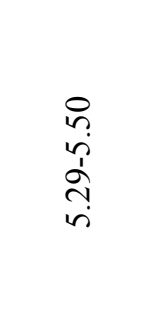 }} & $\begin{array}{l}n \\
i n\end{array}$ \\
\hline & & $\begin{array}{ll}\dot{f} & \stackrel{n}{7} \\
\dot{n} & i n\end{array}$ \\
\hline & & $\begin{array}{cc}m & \vec{m} \\
m & \forall\end{array}$ \\
\hline & $\underset{\forall}{\stackrel{\nabla}{+}}$ & $\stackrel{\vartheta}{\vec{r}}$ \\
\hline & $\begin{array}{l}\text { Oे } \\
+ \\
+\end{array}$ & $\exists$ \\
\hline & ڤे & $\begin{array}{ll}\dot{1} & \infty \\
\stackrel{r}{r} & \stackrel{n}{r}\end{array}$ \\
\hline \multirow{3}{*}{\multicolumn{2}{|c|}{$\begin{array}{l}\hat{n} \\
\stackrel{1}{0} \\
\infty \\
\infty \\
- \\
-\end{array}$}} & $\stackrel{+}{\sim}$ \\
\hline & & $\stackrel{d}{\stackrel{d}{n}} \stackrel{\vec{m}}{i}$ \\
\hline & & 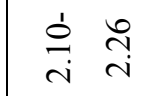 \\
\hline \multirow{5}{*}{\multicolumn{2}{|c|}{ 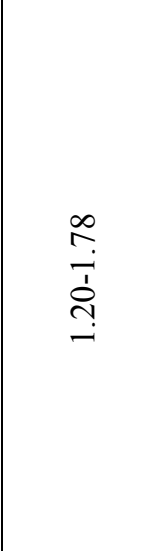 }} & $\stackrel{શ}{I}$ \\
\hline & & 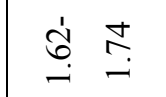 \\
\hline & & 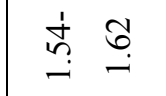 \\
\hline & & $\stackrel{+}{+} \stackrel{n}{\stackrel{n}{-}}$ \\
\hline & & సิ ঙ̊ \\
\hline & $\begin{array}{l}\infty \\
\infty \\
0\end{array}$ & $\begin{array}{l}\infty \\
\infty \\
0\end{array}$ \\
\hline$\sum^{0}$ & : & 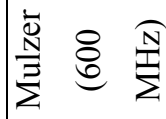 \\
\hline
\end{tabular}

\begin{tabular}{|c|c|}
\hline$\stackrel{\Xi}{\stackrel{\Xi}{I}}$ & $\stackrel{\circ}{\stackrel{ }{I}}$ \\
\hline $\begin{array}{l}\vec{n} \\
\cong\end{array}$ & $\begin{array}{l}\stackrel{0}{+} \\
\stackrel{+}{-}\end{array}$ \\
\hline $\begin{array}{l}\infty \\
i \\
\stackrel{d}{2}\end{array}$ & $\stackrel{\vec{i}}{\stackrel{\imath}{2}}$ \\
\hline さே. & $\begin{array}{l}0 \\
\grave{I}\end{array}$ \\
\hline $\overrightarrow{\mathrm{I}}$ & $\overrightarrow{\mathrm{I}}$ \\
\hline$\stackrel{m}{\stackrel{n}{r}}$ & $\stackrel{9}{\stackrel{2}{\sigma}}$ \\
\hline$\stackrel{\sim}{2}$ & $\stackrel{0}{\ddot{r}}$ \\
\hline$\stackrel{N}{N}$ & $\begin{array}{l}\infty \\
\stackrel{i}{i}\end{array}$ \\
\hline$\vec{n}$ & $\begin{array}{l}0 \\
i n\end{array}$ \\
\hline$\dot{g}$ & $\stackrel{0}{\dot{n}}$ \\
\hline $\begin{array}{l}\stackrel{\circ}{ } \\
\stackrel{f}{f}\end{array}$ & $\begin{array}{l}\infty \\
\stackrel{\mathcal{f}}{f}\end{array}$ \\
\hline $\begin{array}{l}\infty \\
\dot{\rho} \\
\dot{n}\end{array}$ & $\stackrel{0}{i}$ \\
\hline$\stackrel{m}{m}$ & ָे \\
\hline$\stackrel{i}{m}$ & i. \\
\hline ?ֶ. & 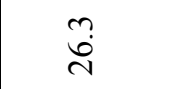 \\
\hline ì & $\stackrel{m}{a}$ \\
\hline$\vec{n}$ & ஸे \\
\hline $\begin{array}{l}\stackrel{\bullet}{\sim} \\
\stackrel{+}{\sim}\end{array}$ & $\stackrel{n}{\sim}$ \\
\hline$\stackrel{n}{\tilde{N}}$ & $\begin{array}{l}0 \\
\text { i }\end{array}$ \\
\hline$\stackrel{\circ}{\dot{\Xi}}$ & $\stackrel{\circ}{\dot{I}}$ \\
\hline 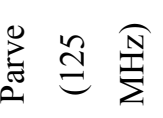 & 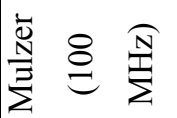 \\
\hline
\end{tabular}

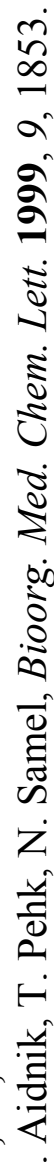

ᄋ̊ำ

空

语

가

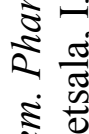

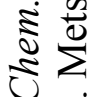

$\exists<$ 


\begin{tabular}{|c|c|c|}
\hline & గn & ñ \\
\hline & $\begin{array}{l}\stackrel{n}{+} \\
i n\end{array}$ & f \\
\hline & $\overrightarrow{\text { స̆ }}$ & $\begin{array}{l}\tilde{N} \\
\text { nn }\end{array}$ \\
\hline & 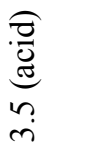 & 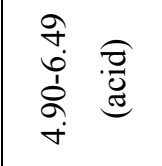 \\
\hline & $\hat{\sim}$ & $\stackrel{n}{\leftrightarrow}$ \\
\hline & $\stackrel{\infty}{\stackrel{\infty}{\infty}}$ & $\underset{\dot{m}}{0}$ \\
\hline & $\stackrel{0}{\stackrel{0}{i}}$ & $\stackrel{\substack{+i}}{i}$ \\
\hline & ते & 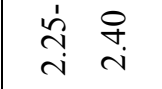 \\
\hline \multirow{2}{*}{\multicolumn{2}{|c|}{$\begin{array}{l}n \\
\\
\tilde{n} \\
\sigma \\
-1\end{array}$}} & $\stackrel{\infty}{a} \frac{\infty}{i}$ \\
\hline & & $\hat{a}$ \\
\hline \multirow{6}{*}{\multicolumn{2}{|c|}{ 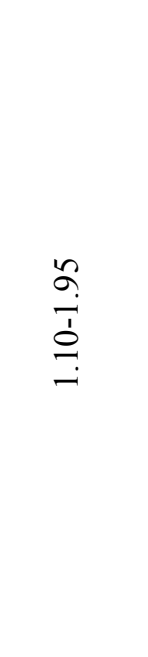 }} & 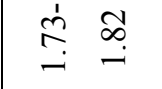 \\
\hline & & $\underset{-}{+} \stackrel{+}{\stackrel{1}{I}}$ \\
\hline & & $\stackrel{n}{n} \underset{\sim}{n}$ \\
\hline & & 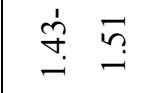 \\
\hline & & ત્่ \\
\hline & & $\stackrel{0}{\longrightarrow}$ \\
\hline & $\begin{array}{l}\infty \\
\infty \\
0 \\
0\end{array}$ & $\stackrel{\infty}{\infty}$ \\
\hline $\begin{array}{c}\stackrel{8}{\tilde{E}} \\
\text { ñ }\end{array}$ & 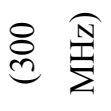 & 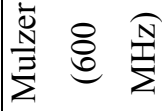 \\
\hline
\end{tabular}

\begin{tabular}{|c|c|c|}
\hline & $\begin{array}{l}\stackrel{0}{\perp} \\
\stackrel{-}{I}\end{array}$ & $\stackrel{\infty}{\stackrel{\infty}{I}}$ \\
\hline & $\stackrel{\check{I}}{\tilde{I}}$ & $\stackrel{\check{I}}{I}$ \\
\hline & $\begin{array}{l}n \\
n \\
n\end{array}$ & $\begin{array}{l}m \\
\stackrel{n}{n} \\
m\end{array}$ \\
\hline & $\begin{array}{l}0 \\
\stackrel{\sim}{-}\end{array}$ & $\stackrel{n}{m}$ \\
\hline & $\stackrel{0}{\stackrel{\Xi}{\beth}}$ & 仓े \\
\hline & 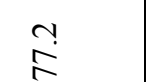 & $\stackrel{ }{\stackrel{2}{ }}$ \\
\hline & $\ddot{n}$ & $\stackrel{\vec{r}}{\sim}$ \\
\hline & $\begin{array}{l}\infty \\
\dot{\varphi} \\
\wp\end{array}$ & ڤें \\
\hline & $\stackrel{\vartheta}{\dot{f}}$ & $\vec{y}$ \\
\hline & $\stackrel{ナ}{\vec{\forall}}$ & $\stackrel{n}{\forall}$ \\
\hline & $\underset{\substack{\infty \\
\infty}}{\infty}$ & $\vec{\infty}$ \\
\hline & $\frac{n}{n}$ & $\underset{m}{\stackrel{+}{n}}$ \\
\hline & $\begin{array}{l}\infty \\
\dot{\sim} \\
\dot{m}\end{array}$ & ఫેे \\
\hline & $\begin{array}{l}\infty \\
\dot{m} \\
\dot{m}\end{array}$ & $\begin{array}{l}\infty \\
\dot{n}\end{array}$ \\
\hline & ڤై & $\stackrel{n}{n}$ \\
\hline & $\frac{0}{m}$ & $\stackrel{i}{\dot{m}}$ \\
\hline & $\begin{array}{l}0 \\
\stackrel{\infty}{N} \\
\stackrel{N}{N}\end{array}$ & 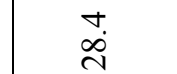 \\
\hline & $\overrightarrow{\sim \vec{n}}$ & $\begin{array}{l}\text { ָे } \\
\text { na }\end{array}$ \\
\hline & $\stackrel{?}{\sim}$ & $\stackrel{n}{+}$ \\
\hline & $\ddot{i}$ & $\begin{array}{l}0 \\
\stackrel{i}{i}\end{array}$ \\
\hline & $\stackrel{\vec{\vartheta}}{\underline{\eta}}$ & $\stackrel{\circ}{\stackrel{\dot{\Xi}}{ }}$ \\
\hline & $\stackrel{\widehat{N}}{\stackrel{N}{\Sigma}}$ & 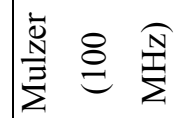 \\
\hline
\end{tabular}

